\title{
Conjugate Addition of 2-Acetylindole Enolates to Unsaturated Oxazolopiperidone Lactams. Enantioselective Access to the Tetracyclic Ring System of Ervitsine
}

\author{
Mercedes Amat,* Begoña Checa, Núria Llor, Maria Pérez, and Joan Bosch
}

Dedicated to Prof. Carmen Nájera on the occasion of her $60^{\text {th }}$ birthday

Keywords: Lactams / Asymmetric synthesis / Cyclization / Michael addition / Heterocycles

\begin{tabular}{|c|c|}
\hline $\begin{array}{l}\text { The stereochemical outcome of the conjugate addition reactions of } \\
\text { 2-acetylindole enolates to unsaturated phenylglycinol-derived } \\
\text { oxazolopiperidone lactams 1a-f is studied. After reduction of the 2- } \\
\text { acylindole carbonyl group, the Michael adduct cis-6 underwent a }\end{array}$ & $\begin{array}{l}\text { Lewis acid-promoted intramolecular } \alpha \text {-amidoalkylation, } \\
\text { enantioselectively leading to the tetracyclic ring system of the } \\
\text { indole alkaloid ervitsine. }\end{array}$ \\
\hline
\end{tabular}

acylindole carbonyl group, the Michael adduct cis-6 underwent a

\section{Introduction}

Phenylglycinol-derived oxazolopiperidone lactams have proven to be versatile scaffolds that allow the regio- and stereocontrolled introduction of substituents at the different positions of the piperidine ring, thus providing access to enantiopure piperidines bearing virtually any type of substitution pattern and also to more complex piperidine-containing alkaloids, including indole alkaloids. ${ }^{[1]}$ In particular, the stereoselective introduction of carbon appendages at the piperidine 4-position by conjugate addition reactions requires the activation of this position, which can be accomplished by generation of a conjugated carbon-carbon double bond taking advantage of the lactam carbonyl group.

In previous work we have exploited the conjugate addition of lower order alkyl- and arylcyanocuprates to unsaturated oxazolopiperidone lactams for the enantioselective synthesis of cis$2,4^{-[2]}$ and cis-3,4-disubstituted piperidines, ${ }^{[3]}$ as well as 2,4bridged $^{[2]}$ and $c i s-3,4$-fused piperidine ${ }^{[4]}$ derivatives, including the antidepressant drug (-)-paroxetine ${ }^{[5]}$ and synthetic intermediates en route to the indole alkaloid (-)-16-episilicine ${ }^{[6]}$ and alkaloids of the madangamine group. ${ }^{[7]}$

Similarly, stereocontrolled conjugate addition reactions of sulfur-stabilized nucleophiles and indoleacetic ester enolates have been successfully employed as the key steps in enantioselective formal syntheses of uleine ${ }^{[8]}$ and Strychnos alkaloids. ${ }^{[9,10]}$

\footnotetext{
Laboratory of Organic Chemistry, Faculty of Pharmacy, and Institute of Biomedicine (IBUB), University of Barcelona, Av. Joan XXIII s/n, 08028 Barcelona (Spain)

Fax: (+34) 934-024-539

E-mail: $\underline{\text { amat@ub.edu }}$
}

\section{Results and Discussion}

We report herein the conjugate addition of 2-acetylindole enolates to unsaturated phenylglycinol-derived oxazolopiperidone lactams and the subsequent construction of the tetracyclic framework of the indole alkaloid ervitsine by closure of the sevenmembered ring by intramolecular $\alpha$-amidoalkylation on the indole 3-position (Scheme 1).

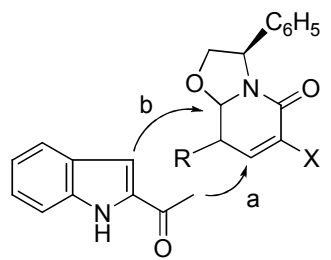

a) Stereoselective conjugate addition (bond formed: $\mathrm{C}^{14}-\mathrm{C}^{15}$ )

b) Intramolecular $\alpha$-amidoalkylation (bond formed: $\mathrm{C}^{5}-\mathrm{C}^{7}$ )

Scheme 1. Synthetic strategy.

As the starting unsaturated lactams we selected lactams 1a-f (Figure 1 and Scheme 2), all of them bearing an ethyl substitutent at the 8-position of the oxazolopiperidone system. The preparation of 3,8a-cis lactams $\mathbf{1 a - d ^ { [ 3 , 6 , 8 ] }}$ has previously been reported. The new 3,8a-trans lactams 1e and $\mathbf{1 f}$ were prepared from the known lactams $\mathbf{2} \mathbf{a}^{[11]}$ and $\mathbf{2} \mathbf{b}^{[11]}$, via the respective seleno derivatives $\mathbf{3 a}$ and $\mathbf{3 b}$, as outlined in Scheme 2.

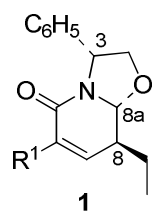

$$
\begin{aligned}
& \text { a } \mathrm{R}^{1}=\mathrm{H} \\
& \text { b } \mathrm{R}^{1}=\mathrm{CO}_{2} \mathrm{Bn} \\
& \text { c } \mathrm{R}^{1}=\mathrm{CO}_{2} \mathrm{Me}
\end{aligned}
$$$$
\mathbf{d ~ R}^{1}=\mathrm{CO}_{2} t \mathrm{Bu}
$$

Figure 1. Unsaturated oxazolopiperidone lactams. 


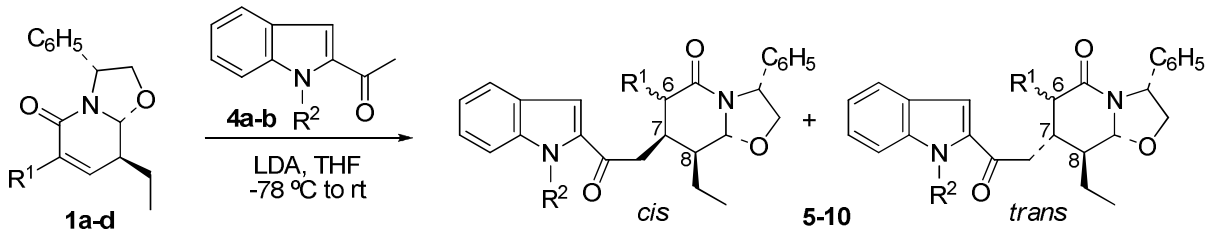

\begin{tabular}{|c|c|c|c|c|c|c|c|c|}
\hline Entry & Lactam & Indole (equiv.) & Time & $\mathbf{R}^{1}$ & $\mathbf{R}^{2}$ & Product & $\begin{array}{c}\mathbf{H}^{7}-\mathbf{H}^{8} \\
\text { cis/trans ratio }\end{array}$ & Yield $(\%)^{[\mathrm{a}]}$ \\
\hline 1 & 1a & $4 \mathbf{a}(1.2)$ & $16 \mathrm{~h}$ & $\mathrm{H}$ & $\mathrm{Me}$ & 5 & $1: 2$ & 24 \\
\hline 2 & $1 \mathbf{a}$ & $4 \mathbf{b}(1.2)$ & $10 \mathrm{~h}$ & $\mathrm{H}$ & $\mathrm{H}$ & 6 & $1: 3$ & 30 \\
\hline 3 & $1 \mathbf{a}$ & $4 b(2.5)$ & $12 \mathrm{~h}$ & $\mathrm{H}$ & $\mathrm{H}$ & 6 & $1: 2.3$ & 56 \\
\hline 6 & $1 \mathbf{b}$ & $4 \mathbf{a}(1.2)$ & $23 \mathrm{~h}$ & $\mathrm{CO}_{2} \mathrm{Bn}$ & $\mathrm{Me}$ & 7 & $3: 1$ & 42 \\
\hline 7 & $1 c$ & $4 \mathbf{b}(1.2)$ & $16 \mathrm{~h}$ & $\mathrm{CO}_{2} \mathrm{Me}$ & $\mathrm{H}$ & 8 & $3: 1$ & 38 \\
\hline 8 & $1 b$ & $4 b(1.2)$ & $16 \mathrm{~h}$ & $\mathrm{CO}_{2} \mathrm{Bn}$ & $\mathrm{H}$ & 9 & $4: 1$ & 46 \\
\hline
\end{tabular}

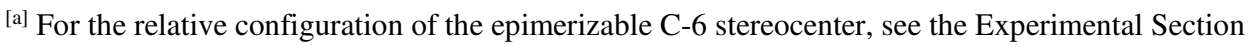

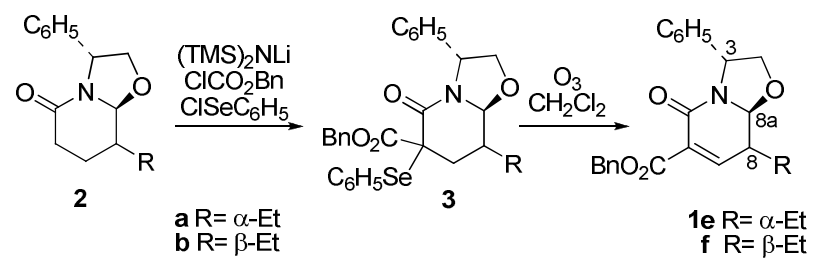

Scheme 2. Preparation of unsaturated lactams 1e and $\mathbf{1 f}$.

Table 1 summarizes the results obtained in the conjugate addition reactions of 2 -acetylindole enolates $\mathbf{4 a}$ and $\mathbf{4 b}$ to unsaturated lactams 1a-d. The initial experiments were carried out using the enolate of 2-acetyl-1-methylindole (4a). Starting from unsaturated lactam 1a, which lacks the activating electronwithdrawing benzyloxycarbonyl group, the use of a slight excess (1.2 equiv.) of the nucleophile (entry 1 ) led to the expected adducts 5 in low yield (24\%) as a 1:2 epimeric mixture of C-7/C-8 cis/trans isomers. A similar result was observed using the enolate of 2acetylindole (4b): compound $\mathbf{6}$ was obtained in $30 \%$ yield as a C$7 / \mathrm{C}-8$ cis/trans epimeric mixture in a $1: 3$ ratio (entry 2 ). The yield grew progressively higher as the excess of the nucleophile was increased (entries 3 and 4), reaching $68 \%$ when using 5 equiv. of enolate.

The relative configuration of the cis and trans isomers was evident from the shielding of the oxazolopiperidone 6-carbon in the ${ }^{13} \mathrm{C}$ NMR spectrum of $c i s-6$ ( $\delta 37.9 \mathrm{ppm}$; compare with $\delta 42.0 \mathrm{ppm}$ in trans-6) exerted by the axial ethyl substituent at C-8.

The facial selectivity of the conjugate addition reactions to lactam 1a $\left(\mathrm{R}^{1}=\mathrm{H}\right)$ can be accounted for by considering that the addition of stabilized anions to $\alpha, \beta$-unsaturated carbonyl compounds is a reversible process that in the case of 5-substituted 5,6-dihydro-2-pyridones leads to the thermodynamically more stable trans-4,5-disubstituted derivative. ${ }^{[12]}$ Accordingly, in the reaction of lactam 1a with acetylindole $4 \mathbf{b}$ an increase of the ratio of the isomer cis-6 was observed when the reaction was quenched after a short reaction time (Table 1, entry 5).

In clear contrast from the stereochemical standpoint, similar conjugate addition reactions from the activated lactams $\mathbf{1 b - d}$, which bear an additional activating alcoxycarbonyl substituent, led to the respective adducts $\mathbf{7 - 1 0}$ as diastereoisomeric mixtures in which the $\mathrm{C}-7 / \mathrm{C}-8$ cis isomers predominated (entries 6-10). ${ }^{[13]}$ As in the above lactam 1a, the use of a 5 equiv. excess of nucleophile in these reactions resulted in higher yields, lactams $\mathbf{9}$ and $\mathbf{1 0}$ being formed in $90 \%$ (compare entries 8 and 9) and $87 \%$ yield (entry 10), respectively, even after shorter reaction times.

The C-7/C-8 cis relative configuration in the major isomer cis-9 was confirmed by its conversion $\left[\mathrm{H}_{2}, \mathrm{Pd}(\mathrm{OH})_{2}\right.$; then toluene at reflux] to cis-6, the minor isomer obtained from lactam 1a (Scheme 3).

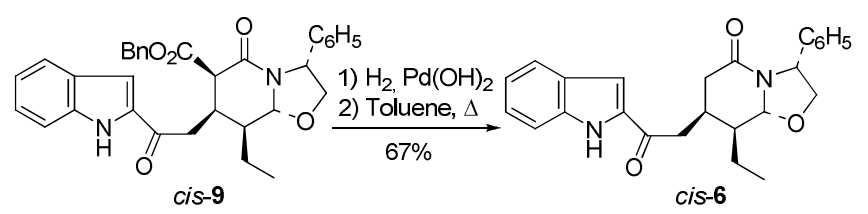

Scheme 3. Removal of the benzyloxycarbonyl substituent.

On the other hand, the predominance of the cis isomer in the conjugate addition to lactams $\mathbf{1 b}-\mathbf{d}\left(\mathrm{R}^{1}=\mathrm{CO}_{2} \mathrm{R}\right)$ can be rationalized by considering that in these cases the equilibration 
takes place to a lesser extent as a consequence of the higher stability of the initially formed adduct (a 1,3-dicarbonyl enolate). The process would then occur mainly under stereoelectronic control, ${ }^{[14]}$ which involves an axial approach of the nucleophile to the electrophilic carbon of the conjugate double bond from the exo face of the bicyclic system as depicted in Figure 2. In fact, irreversible conjugate additions (for instance, of organocuprates) to unsaturated oxazolopiperidone lactams (1a-f or related lactams) have been reported to occur under stereoelectronic control with complete exo facial selectivity. ${ }^{[15]}$

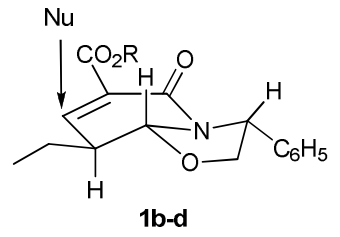

$$
\begin{aligned}
\text { 1e } \mathrm{R}^{1}=\mathrm{H} ; \mathrm{R}^{2}=\mathrm{Et} \\
\text { 1f } \mathrm{R}^{1}=\mathrm{Et} ; \mathrm{R}^{2}=\mathrm{H}
\end{aligned}
$$

Figure 2. Stereoelectronic control in the conjugate addition.

A similar stereoelectronically controlled facial stereoselectivity was observed in the conjugate addition of the enolate of $\mathbf{4 b}$ to the C-8 epimeric 3,8a-trans lactams 1e and 1f (Figure 2), the respective exo adducts cis-11 and trans-12 being formed as the major isomers (Scheme 4).

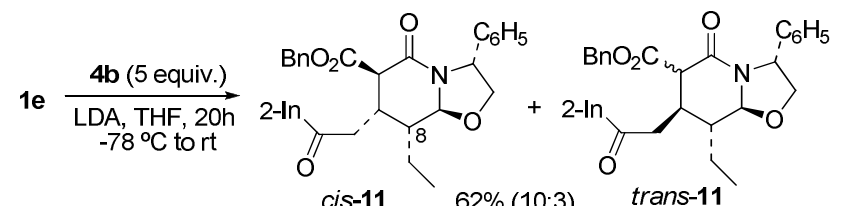

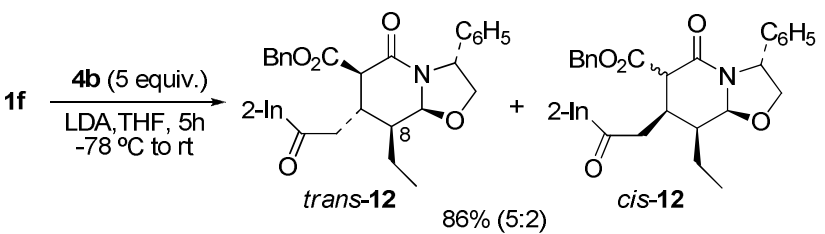

Scheme 4. Conjugate addition reactions of 2-acetylindole to unsaturated lactams $\mathbf{1 e}$ and $\mathbf{1 f}$; In= indolyl.

To study the closure of the seven-membered ring characteristic of ervitsine, we initially selected lactam trans-6. However, all attempts to promote the cyclization under a variety of acidic conditions $\left(\mathrm{TiCl}_{4}, \mathrm{CH}_{2} \mathrm{Cl}_{2}\right.$, reflux; $\mathrm{BF}_{3} \cdot \mathrm{Et}_{2} \mathrm{O}, \mathrm{CH}_{2} \mathrm{Cl}_{2}$, reflux; $\mathrm{HCl}$, $\mathrm{MeOH}$ or $\left.\mathrm{C}_{6} \mathrm{H}_{6}\right)$ resulted in failure. Enamide $\mathbf{1 3}^{[16]}$ and the $8 \mathrm{a}-$ epimer (14) and the 8,8a-diastereoisomer (15) of trans-6 were the only isolable products (Scheme 5). ${ }^{[17]}$ The stereochemistry of $\mathbf{1 4}$ and $\mathbf{1 5}$ was confirmed when these compounds were unambiguously prepared from trans-12 and cis-11, respectively, by debenzylation $\left[\mathrm{H}_{2}, \mathrm{Pd}(\mathrm{OH})_{2}\right]$ followed by decarboxylation.

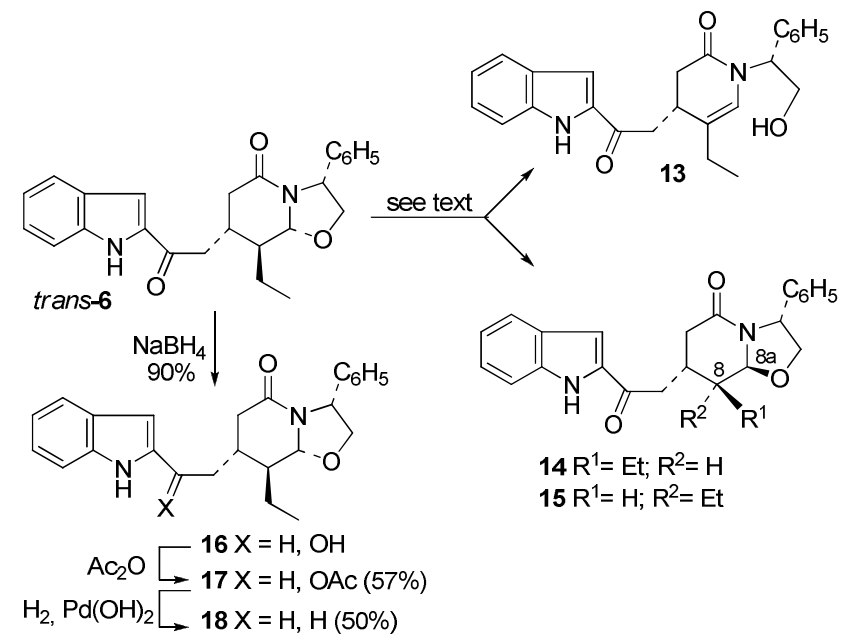

Scheme 5.

Taking into account that the isolation of compounds 13-15 clearly indicated that the $N$-acyl iminium cation ${ }^{[18]}$ had been formed, the failure of the cyclization was attributed to the deactivating effect of the carbonyl group conjugated with the indole ring. For this reason, acylindole trans-6 was converted to alcohol 16 and then to the indolylethyl derivative 18 by hydrogenolysis of the corresponding acetate $\mathbf{1 7}$.

The desired cyclization upon the indole 3-position did not occur from 16 or 18 either, under a variety of acidic conditions, only extensive decomposition being observed.

Bearing in mind that the closure of the seven-membered ring of ervitsine and analogs has been successfully achieved by a related intramolecular iminium ion cyclization, ${ }^{[19]}$ at this point we reasoned that conformational factors could be responsible for the reluctance of the above C-7/C-8 trans derivatives to undergo intramolecular $\alpha$-amidoalkylation: cyclization would involve an encumbered conformation in which both C-7 and C-8 substituents should be axial. To confirm this hypothesis, we decided to study related $\alpha$-amidoalylation reactions from $\mathrm{C}-7 / \mathrm{C}-8$ cis lactams.

However, no cyclized products were detected upon treatment of cis-6 with $\mathrm{TiCl}_{4}$, the $8 \mathrm{a}$-epimer of cis-6 being the only isolable product. ${ }^{[20]}$ As in the above C-7/C-8 trans series, 2-acylindole cis-6 was reduced to alcohol 19, and then converted to indolylethyl derivative $\mathbf{2 1}$ via the corresponding acetate $\mathbf{2 0}$ (Scheme 6).
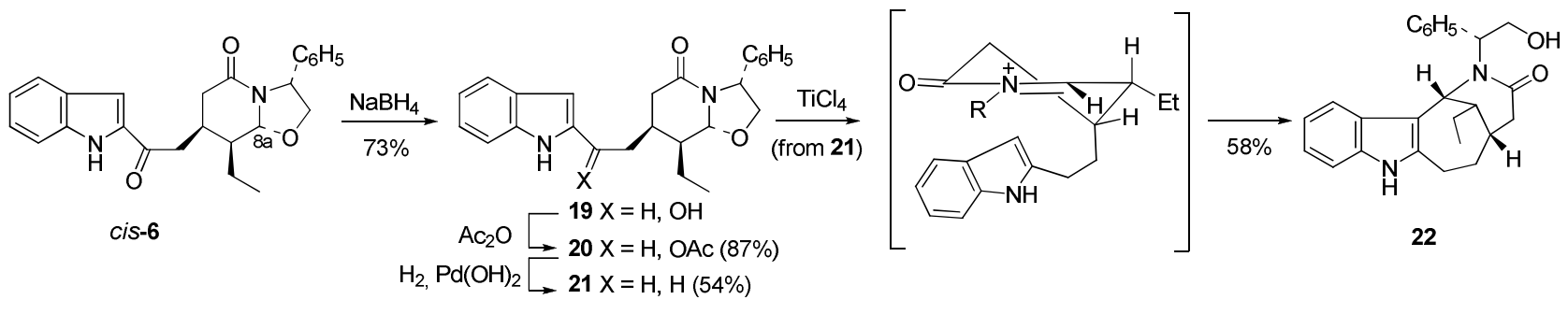

Scheme 6. Access to the tetracyclic ring system of ervitsine. 
Although alcohol 19 underwent extensive decomposition upon acidic treatment, to our delight, when the reduced derivative $\mathbf{2 1}$ was treated with $\mathrm{TiCl}_{4}$ in refluxing $\mathrm{CH}_{2} \mathrm{Cl}_{2}$, tetracycle 22 was isolated in $58 \%$ yield.

\section{Conclusions}

In conclusion, conjugate addition reactions of 2-acetylindole enolates to phenylglycinol-derived unsaturated $\delta$-lactams allow the stereocontrolled formation of $\mathrm{C}-\mathrm{C}$ bonds at the piperidine 4position. Depending on the absence or presence of an additional electron-withdrawing substituent conjugated with the $\mathrm{C}-\mathrm{C}$ double bond, the reaction predominantly leads to either trans- or cis-4,5disubtituted enantiopure 2-piperidone derivatives, respectively.

The synthetic potential of the resulting Michael adducts has been demonstrated with the enantioselective construction of the tetracyclic ring system of ervitsine, a minor indole alkaloid isolated from Pandaca boiteaui ${ }^{[21]}$ that lacks the characteristic tryptamine moiety present in most monoterpenoid indole alkaloids. Starting from an appropriate lactam bearing a C-8 substituent precursor of the exocyclic methylene group, the strategy developed here, involving a stereoselective conjugate addition and an intramolecular $\alpha$-amidoalkylation as the key steps (see Scheme 1), may be applied to the enantioselective synthesis of ervitsine. ${ }^{[22]}$

\section{Experimental Section}

General: Flash chromatography was carried out on $\mathrm{SiO}_{2}$ (silica gel 60, SDS, 0.04-0.06 mm) or (when indicated) using a cartridge containing amine functionalized silica. Optical rotations were measured on Perkin-Elmer 241 polarimeter. High resolution mass spectra (HMRS; LC/MSD TOF Agilent Technologies) were performed by Serveis Científico-Tècnics, Barcelona. Microanalyses (Carlo Erba 1106 analyzer) were performed by Centre d'Investigació $i$ Desenvolupament (CSIC), Barcelona. Only noteworthy IR absorptions ( $\mathrm{cm}^{-1}$; Perkin-Elmer 1600) are listed. NMR spectra were recorded at $200,300,400$ or $500 \mathrm{MHz}\left({ }^{1} \mathrm{H}\right)$ and $75.4,100.6$ or $125.9 \mathrm{MHz}\left({ }^{13} \mathrm{C}\right)$.

\section{(3R,8R,8aS)-6-(Benzyloxycarbonyl)-8-ethyl-5-oxo-3-phenyl-6-} (phenylselenyl)-2,3,6,7,8,8a-hexahydro-5H-oxazolo[3,2-

a]pyridine (3a): Lithium bis(trimethylsilyl)amide $(9.4 \mathrm{~mL}$ of a 1.0 $\mathrm{M}$ solution in THF, $9.4 \mathrm{mmol}$ ) was slowly added at $-78{ }^{\circ} \mathrm{C}$ to a solution of lactam $2 \mathrm{a}^{[11]}(1.08 \mathrm{~g}, 4.24 \mathrm{mmol})$ in anhydrous THF (50 $\mathrm{mL}$ ), and the resulting mixture was stirred for $90 \mathrm{~min}$. Then, benzyl chloroformate $(710 \mu \mathrm{L}, 4.24 \mathrm{mmol})$ and, after $2 \mathrm{~h}$ of continuous stirring at $-78{ }^{\circ} \mathrm{C}$, a solution of $\mathrm{C}_{6} \mathrm{H}_{5} \mathrm{SeCl}(1.14 \mathrm{~g}, 5.09 \mathrm{mmol})$ in anhydrous THF ( $5 \mathrm{~mL}$ ) were added. The mixture was stirred for 2 $\mathrm{h}$ and poured into saturated aqueous $\mathrm{NH}_{4} \mathrm{Cl}$. The aqueous layer was extracted with EtOAc, and the combined organic extracts were dried and concentrated. Flash chromatography (hexane to 4:1 hexane-EtOAc) of the resulting oil afforded the corresponding selenides 3a as a mixture of C-6 epimers $(1.40 \mathrm{~g}, 62 \%)$. Data for 3a: Orange oil. ${ }^{1} \mathrm{H}$ NMR $\left(300 \mathrm{MHz}, \mathrm{CDCl}_{3}, 25^{\circ} \mathrm{C}\right): \delta=0.74(\mathrm{t}, J=$ $7.5 \mathrm{~Hz}, 3 \mathrm{H}, \mathrm{CH}_{3}$ ethyl), 0.80 (t, $J=7.3 \mathrm{~Hz}, 3 \mathrm{H}, \mathrm{CH}_{3}$ ethyl), $1.12-$ $1.18\left(\mathrm{~m}, 1 \mathrm{H}, \mathrm{CH}_{2}\right.$ ethyl), 1.12-1.22 (m, $1 \mathrm{H}, \mathrm{CH}_{2}$ ethyl), 1.57-1.95 (m, 3H, $\mathrm{CH}_{2}$ ethyl, H-7, H-8), 1.61-1.72 (m, 2H, $\mathrm{CH}_{2}$ ethyl, H-8), 1.83-2.00 (m, 2H, H-7), 2.33 (dd, $J=13.8,2.1 \mathrm{~Hz}, 1 \mathrm{H}, \mathrm{H}-7), 3.71$ (dd, $J=9.0,7.8 \mathrm{~Hz}, 1 \mathrm{H}, \mathrm{H}-2), 3.78$ (dd, $J=9.0,7.5 \mathrm{~Hz}, 1 \mathrm{H}, \mathrm{H}-2$ ),
4.34 (d, $J=8.1 \mathrm{~Hz}, 1 \mathrm{H}, \mathrm{H}-8 \mathrm{a}), 4.37$ (dd, $J=9.0,7.5 \mathrm{~Hz}, 1 \mathrm{H}, \mathrm{H}-2)$, 4.46 (dd, $J=9.0,7.8 \mathrm{~Hz}, 1 \mathrm{H}, \mathrm{H}-2), 4.67$ (d, $J=7.8 \mathrm{~Hz}, 1 \mathrm{H}, \mathrm{H}-8 \mathrm{a})$, $5.20\left(\mathrm{~d}, J=6.9 \mathrm{~Hz}, 1 \mathrm{H}, \mathrm{CH}_{2} \mathrm{C}_{6} \mathrm{H}_{5}\right), 5.20\left(\mathrm{~s}, 2 \mathrm{H}, \mathrm{CH}_{2} \mathrm{C}_{6} \mathrm{H}_{5}\right), 5.24(\mathrm{~d}$, $\left.J=6.9 \mathrm{~Hz}, 1 \mathrm{H}, \mathrm{CH}_{2} \mathrm{C}_{6} \mathrm{H}_{5}\right), 5.25(\mathrm{~d}, J=7.8 \mathrm{~Hz}, 1 \mathrm{H}, \mathrm{H}-3), 5.30$ (t, $J$ $=7.5 \mathrm{~Hz}, 1 \mathrm{H}, \mathrm{H}-3), 7.18-7.70(\mathrm{~m}, 30 \mathrm{H}, \mathrm{ArH}) \mathrm{ppm} .{ }^{13} \mathrm{C} \mathrm{NMR}$ $\left(100.6 \mathrm{MHz}, \mathrm{CDCl}_{3}, 25^{\circ} \mathrm{C}\right): \delta=10.5,10.7\left(\mathrm{CH}_{3}\right.$ ethyl), 23.7, 24.0 ( $\mathrm{CH}_{2}$ ethyl), 32.5, 35.2 (C-7), 37.8, 39.3 (C-8), 54.2, 55.6 (C-6), 58.8 (C-3), 67.7, $67.8\left(\mathrm{CH}_{2} \mathrm{C}_{6} \mathrm{H}_{5}\right), 72.1,72.3(\mathrm{C}-2), 92.0,92.3$ (C8a), 125.7, 126.1 (C-o), 126.6, 126.7 (C-m), 127.3, 127.5 (C-p), 127.9, 128.0 (C-o), 128.0, 128.1 (C-p), 128.3, 128.4 (C-m), 128.5, $128.6(\mathrm{C}-o), 128.7,128.8(\mathrm{C}-m), 129.3,129.5(\mathrm{C}-i), 135.0,135.1$ (C-i), 138.0 (C-p), 138.5, 138.7 (C-i), 164.5, 164.9 (NCO), 169.9, 170.9 (COO) ppm.

(3R,8S,8aS)-6-(Benzyloxycarbonyl)-8-ethyl-5-oxo-3-phenyl-6(phenylselenyl)-2,3,6,7,8,8a-hexahydro-5H-oxazolo[3,2-

a]pyridine (3b): Operating as in the above preparation of $\mathbf{3 a}$, from lactam $2 \mathbf{b}^{[11]}(2 \mathrm{~g}, 7.90 \mathrm{mmol})$ in THF $(100 \mathrm{~mL})$, LiHMDS $(17.3$ $\mathrm{mL}$ of a 1.0 M solution in THF), benzyl chloroformate $(1.2 \mathrm{~mL}, 8.6$ $\mathrm{mmol})$, and $\mathrm{C}_{6} \mathrm{H}_{5} \mathrm{SeCl}(1.70 \mathrm{~g}, 8.80 \mathrm{mmol})$ in THF $(20 \mathrm{~mL})$, a mixture of C-6 epimeric selenides (3.46 g, 82\%) was obtained. Pure isomers were isolated after a subsequent flash chromatography (hexane to 9:1 hexane-EtOAc). Data for $\mathbf{3 b}$ (higher $\mathrm{R}_{f}$ epimer): Orange oil; $[\alpha]^{22} \mathrm{D}=-84.0\left(c=0.5, \mathrm{CHCl}_{3}\right)$. IR (film): $v=1664,1724 \mathrm{~cm}^{-1} .{ }^{1} \mathrm{H}$ NMR $\left(300 \mathrm{MHz}, \mathrm{CDCl}_{3}, 25^{\circ} \mathrm{C}\right): \delta$ $=0.82\left(\mathrm{t}, J=7.5 \mathrm{~Hz}, 3 \mathrm{H}, \mathrm{CH}_{3}\right.$ ethyl), $1.29-1.38\left(\mathrm{~m}, 1 \mathrm{H}, \mathrm{CH}_{2}\right.$ ethyl), 1.53-1.63 (m, $1 \mathrm{H}, \mathrm{CH}_{2}$ ethyl), 1.72-1.81 (m, 1H, H-8), 1.88-2.02 (m, 1H, H-7), 1.99 (dd, $J=12.9,5.1 \mathrm{~Hz}, 1 \mathrm{H}, \mathrm{H}-7), 3.73$ (dd, $J=$ 8.7, $6.9 \mathrm{~Hz}, 1 \mathrm{H}, \mathrm{H}-2), 4.45$ (t, $J=8.7 \mathrm{~Hz}, 1 \mathrm{H}, \mathrm{H}-2), 4.60$ (d, $J=5.4$ $\mathrm{Hz}, 1 \mathrm{H}, \mathrm{H}-8 \mathrm{a}), 5.09$ (d, $\left.J=12.3 \mathrm{~Hz}, 1 \mathrm{H}, \mathrm{CH}_{2} \mathrm{C}_{6} \mathrm{H}_{5}\right), 5.32$ (d, $J=$ $\left.12.3 \mathrm{~Hz}, 1 \mathrm{H}, \mathrm{CH}_{2} \mathrm{C}_{6} \mathrm{H}_{5}\right), 5.56(\mathrm{t}, J=8.1 \mathrm{~Hz}, 1 \mathrm{H}, \mathrm{H}-3), 7.13-7.55$ (m, 15H, ArH) ppm. ${ }^{13} \mathrm{C}$ NMR (75.4 MHz, $\left.\mathrm{CDCl}_{3}, 25^{\circ} \mathrm{C}\right): \delta=11.4$ $\left(\mathrm{CH}_{3}\right.$ ethyl), 23.3 ( $\mathrm{CH}_{2}$ ethyl), 33.6 (C-7), 35.9 (C-8), $54.2(\mathrm{C}-6)$, 58.1 (C-3), $67.4\left(\mathrm{CH}_{2} \mathrm{C}_{6} \mathrm{H}_{5}\right), 70.8$ (C-2), 87.8 (C-8a), 125.6 (C-o), 126.7 (C-m), 127.3 (C-p), 128.1 (C-o), 128.2 (C-m), 128.4 (C-p), $128.6(\mathrm{C}-o), 129.5(\mathrm{C}-m), 135.2(\mathrm{C}-p), 138.5(\mathrm{C}-i), 139.5(\mathrm{C}-i)$, 167.1 (NCO), 169.4 (COO) ppm. $\mathrm{C}_{29} \mathrm{H}_{29} \mathrm{NO}_{4} \mathrm{Se}$ (534.51): calcd. C 65.17, H 5.47, N 2.62; found C 65.19, H 5.58, N 2.54. Data for $3 \mathbf{b}$ (lower $\mathrm{R}_{f}$ epimer): Orange oil; $[\alpha]^{22} \mathrm{D}=-22.0\left(c=0.5, \mathrm{CHCl}_{3}\right)$. IR (film): $v=1661,1732 \mathrm{~cm}^{-1} .{ }^{1} \mathrm{H} \mathrm{NMR}\left(300 \mathrm{MHz}, \mathrm{CDCl}_{3}, 25^{\circ} \mathrm{C}\right): \delta$ $=0.81\left(\mathrm{t}, J=7.5 \mathrm{~Hz}, 3 \mathrm{H}, \mathrm{CH}_{3}\right.$ ethyl $), 1.12-1.23\left(\mathrm{~m}, 1 \mathrm{H}, \mathrm{CH}_{2}\right.$ ethyl $)$, 1.55-1.67 (m, 1H, $\mathrm{CH}_{2}$ ethyl), 2.04 (dd, $J=15.0,4.2 \mathrm{~Hz}, 1 \mathrm{H}, \mathrm{H}-7$ ), 2.22-2.28 (m, 1H, H-8), 2.56 (dd, $J=15.0,6.6 \mathrm{~Hz}, 1 \mathrm{H}, \mathrm{H}-7), 3.84$ (dd, $J=8.7,6.3 \mathrm{~Hz}, 1 \mathrm{H}, \mathrm{H}-2), 4.34$ (dd, $J=8.7,7.5 \mathrm{~Hz}, 1 \mathrm{H}, \mathrm{H}-2)$, 4.85 (d, $J=4.8 \mathrm{~Hz}, 1 \mathrm{H}, \mathrm{H}-8 \mathrm{a}), 5.18$ (s, $\left.2 \mathrm{H}, \mathrm{CH}_{2} \mathrm{C}_{6} \mathrm{H}_{5}\right), 5.35$ (t, $J=$ $6.8 \mathrm{~Hz}, 1 \mathrm{H}, \mathrm{H}-3), 7.06-7.47$ (m, 15H, ArH) ppm. ${ }^{13} \mathrm{C}$ NMR $(75.4$ $\left.\mathrm{MHz}, \mathrm{CDCl}_{3}, 25^{\circ} \mathrm{C}\right): \delta=11.6\left(\mathrm{CH}_{3}\right.$ ethyl $), 18.7\left(\mathrm{CH}_{2}\right.$ ethyl $), 32.5$ $(\mathrm{C}-7), 37.2(\mathrm{C}-8), 52.7(\mathrm{C}-6), 58.6(\mathrm{C}-3), 67.9\left(\mathrm{CH}_{2} \mathrm{C}_{6} \mathrm{H}_{5}\right), 71.6(\mathrm{C}-$ 2), 89.0 (C-8a), 126.5 (C-o), 126.7 (C-m), 127.6 (C-p), 128.1 (C-o), 128.4 (CHAr), 128.7 (CHAr), 129.4 (CHAr), 135.2 (C-i), 137.7 (C-i), 139.2 (C-i), 165.1 (NCO), 170.2 (COO) ppm. $\mathrm{C}_{29} \mathrm{H}_{29} \mathrm{NO}_{4} \mathrm{Se}$ (534.51): calcd. C 65.17, H 5.47, N 2.62; found C 64.81, H 5.64, N 2.64 .

(3R,8R,8aS)-6-(Benzyloxycarbonyl)-8-ethyl-5-oxo-3-phenyl3,5,8,8a-tetrahydro-5H-oxazolo $[3,2-a]$ pyridine (1e): A stream of ozone gas was bubbled through a cooled $\left(-78{ }^{\circ} \mathrm{C}\right)$ solution of the selenides 3a (161 mg, $0.30 \mathrm{mmol})$ in anhydrous $\mathrm{CH}_{2} \mathrm{Cl}_{2}(50 \mathrm{~mL})$ until it turned pale blue ( $5 \mathrm{~min}$ ). Then, the solution was purged with $\mathrm{O}_{2}$, and the temperature was slowly raised to $25{ }^{\circ} \mathrm{C}$. After 30 
min of stirring, the mixture was poured intro brine, and the aqueous layer was extracted with $\mathrm{CH}_{2} \mathrm{Cl}_{2}$. The combined organic extracts were dried and concentrated to give unsaturated lactam 1e, which due to its instability was used in the next reaction without further purification. Data for 1e: Yellow oil. ${ }^{1} \mathrm{H}$ NMR $\left(300 \mathrm{MHz}, \mathrm{CDCl}_{3}\right.$, $\left.25^{\circ} \mathrm{C}\right): \delta=1.10\left(\mathrm{t}, J=7.5 \mathrm{~Hz}, 3 \mathrm{H}, \mathrm{CH}_{3}\right.$ ethyl), 1.57-1.66 (m, $1 \mathrm{H}$, $\mathrm{CH}_{2}$ ethyl), 1.81-1.90 (m, 1H, CH 2 ethyl), 2.53-2.61 (m, 1H, H-8), $3.94(\mathrm{dd}, J=9.0,6.0 \mathrm{~Hz}, 1 \mathrm{H}, \mathrm{H}-2), 4.44(\mathrm{dd}, J=9.0,6.9 \mathrm{~Hz}, 1 \mathrm{H}$, $\mathrm{H}-2), 5.10(\mathrm{~d}, J=9.3 \mathrm{~Hz}, 1 \mathrm{H}, \mathrm{H}-8 \mathrm{a}), 5.26(\mathrm{~d}, J=5.3 \mathrm{~Hz}, 1 \mathrm{H}$, $\left.\mathrm{CH}_{2} \mathrm{C}_{6} \mathrm{H}_{5}\right), 5.29(\mathrm{~m}, 1 \mathrm{H}, \mathrm{H}-3), 5.30\left(\mathrm{~d}, J=5.3 \mathrm{~Hz}, 1 \mathrm{H}, \mathrm{CH}_{2} \mathrm{C}_{6} \mathrm{H}_{5}\right)$, 7.19-7.40 (m, 11H, H-7, ArH) ppm.

(3R,8S,8aS)-6-(Benzyloxycarbonyl)-8-ethyl-5-oxo-3-phenyl3,5,8,8a-tetrahydro-5 $\mathrm{H}$-oxazolo[3,2-a]pyridine (1f): Operating as in the above preparation of $\mathbf{1 e}$, from a mixture of selenides $\mathbf{3 b}$ (246 mg, $0.46 \mathrm{mmol})$ in $\mathrm{CH}_{2} \mathrm{Cl}_{2}(15 \mathrm{~mL})$, unsaturated lactam 1f (173 mg, $0.46 \mathrm{mmol}$ ) was obtained, which due to instability was used in the next reaction without further purification. Data for $\mathbf{1 f}$ : Orange oil. ${ }^{1} \mathrm{H}$ NMR $\left(300 \mathrm{MHz}, \mathrm{CDCl}_{3}, 25^{\circ} \mathrm{C}\right): \delta=0.99(\mathrm{t}, J=7.5$ $\mathrm{Hz}, 3 \mathrm{H}, \mathrm{CH}_{3}$ ethyl), 1.38-1.48 (m, $1 \mathrm{H}, \mathrm{CH}_{2}$ ethyl), 1.83-1.92 (m, $1 \mathrm{H}, \mathrm{CH}_{2}$ ethyl), 2.76-2.81 (m, 1H, H-8), 4.00 (dd, $J=8.4,5.7 \mathrm{~Hz}$, $1 \mathrm{H}, \mathrm{H}-2), 4.47$ (dd, $J=8.4,6.9 \mathrm{~Hz}, 1 \mathrm{H}, \mathrm{H}-2), 5.22-5.26$ (m, 3H, $\left.\mathrm{CH}_{2} \mathrm{C}_{6} \mathrm{H}_{5}, \mathrm{H}-3\right), 5.50$ (d, $\left.J=5.4 \mathrm{~Hz}, 1 \mathrm{H}, \mathrm{H}-8 \mathrm{a}\right), 7.24-7.62(\mathrm{~m}, 11 \mathrm{H}$, $\mathrm{H}-7, \mathrm{ArH}) \mathrm{ppm}$.

General Procedure for the Conjugate Addition Reactions: LDA was added to a cooled $\left(-78{ }^{\circ} \mathrm{C}\right)$ solution of 2-acetylindole $(\mathbf{4 a}$ or 4b) in THF, and the mixture was stirred at this temperature for $1 \mathrm{~h}$. Then, a solution of unsaturated lactam $\mathbf{1}$ in THF was added to the solution $\left(-78^{\circ} \mathrm{C}\right)$. The resulting mixture was stirred at room temperature until the disappearance of the starting material was observed by TLC. The reaction was quenched by the addition of saturated aqueous $\mathrm{NH}_{4} \mathrm{Cl}$, and the mixture was extracted with EtOAc. The combined extracts were dried and concentrated to give a residue, which was purified by chromatography.

(3R,7R,8S,8aR)-8-Ethyl-7-[2-(1-methyl-2-indolyl)-2-oxoethyl]5-oxo-3-phenyl-2,3,6,7,8,8a-hexahydro-5 $\mathrm{H}$-oxazolo[3,2-

a]pyridine (trans-5) and its $7 \mathrm{~S}$ epimer (cis-5): Operating as described in the general procedure, from lactam $\mathbf{1 a}^{[3 \mathrm{~b}, 8]}(200 \mathrm{mg}$, $0.82 \mathrm{mmol})$ in THF $(30 \mathrm{~mL})$, LDA $(0.65 \mathrm{~mL}$ of a solution $1.5 \mathrm{M}$ in cyclohexane, $0.98 \mathrm{mmol})$, and a solution of indole $4 \mathrm{a}(170 \mathrm{mg}$, $0.98 \mathrm{mmol}$ ) in $30 \mathrm{~mL}$ of THF for $16 \mathrm{~h}$, compound 5 was obtained as a mixture of C-7 epimers. Flash chromatography (from 1:1 to 3:7 hexane-EtOAc) afforded trans-5 (53 mg, 16\%) and cis-5 (27 $\mathrm{mg}, 8 \%)$. Data for trans-5: Yellow foam; $[\alpha]^{22} \mathrm{D}=-13.0(c=0.5$, $\mathrm{CHCl}_{3}$ ). IR (film): $v=1644,1661 \mathrm{~cm}^{-1} .{ }^{1} \mathrm{H}$ NMR $(400 \mathrm{MHz}$, $\left.\mathrm{CDCl}_{3}, 25{ }^{\circ} \mathrm{C}\right): \delta=1.07$ (t, $J=7.4 \mathrm{~Hz}, 3 \mathrm{H}, \mathrm{CH}_{3}$ ethyl), $1.65-1.89$ (m, 3H, $\mathrm{CH}_{2}$ ethyl, H-8), 2.16 (dd, $J=18.2,9.2 \mathrm{~Hz}, 1 \mathrm{H}, \mathrm{H}-6$ ), 2.502.60 (m, 2H, H-6, H-7), 2.87 (dd, $J=16.2,7.6 \mathrm{~Hz}, 1 \mathrm{H}, \mathrm{CH}_{2} \mathrm{CO}$ ), $3.10\left(\mathrm{dd}, J=16.2,5.2 \mathrm{~Hz}, 1 \mathrm{H}, \mathrm{CH}_{2} \mathrm{CO}\right), 4.05$ (s, $\left.3 \mathrm{H}, \mathrm{NCH}_{3}\right), 4.09$ (d, $J=9.0 \mathrm{~Hz}, 1 \mathrm{H}, \mathrm{H}-2), 4.18$ (dd, $J=9.0,6.8 \mathrm{~Hz}, 1 \mathrm{H}, \mathrm{H}-2), 4.73$ (d, $J=7.6 \mathrm{~Hz}, 1 \mathrm{H}, \mathrm{H}-8 \mathrm{a}), 4.96$ (dd, $J=6.8,1.2 \mathrm{~Hz}, 1 \mathrm{H}, \mathrm{H}-3), 7.11$ (s, 1H, H-3 ind), 7.13-7.20 (m, 1H, H-7 ind), 7.26-7.40 (m, 7H, H5, H-6 ind, ArH), 7.67 (dd, $J=8.2,1.2 \mathrm{~Hz}, 1 \mathrm{H}, \mathrm{H}-4$ ind) ppm. ${ }^{13} \mathrm{C}$ NMR (100.6 MHz, $\left.\mathrm{CDCl}_{3}, 25{ }^{\circ} \mathrm{C}\right): \delta=10.2\left(\mathrm{CH}_{3}\right.$ ethyl), $22.7\left(\mathrm{CH}_{2}\right.$ ethyl), 32.2 (C-7), 37.5 (C-6), $43.7\left(\mathrm{CH}_{2} \mathrm{CO}\right), 44.8$ (C-8), 58.4 (C3), 73.9 (C-2), 91.1 (C-8a), 110.3 (C-3 ind), 111.8 (C-7 ind), 120.7 (C-4 ind), 122.9 (C-6 ind), 125.6 (C-5 ind), 126.2 (C-2 ind), 126.7 (C-o), 128.6 (C-m), 127.6 (C-p), 134.7 (C-i), 140.2 (C-3a ind),
141.2 (C-7a ind), 166.7 (NCO), 192.0 (CO) ppm. HRMS calcd. for $\mathrm{C}_{26} \mathrm{H}_{29} \mathrm{~N}_{2} \mathrm{O}_{3}[\mathrm{M}+\mathrm{H}]^{+}$: 417.2178; found 417.2172. Data for cis-5: Yellow foam. ${ }^{1} \mathrm{H}$ NMR $\left(200 \mathrm{MHz}, \mathrm{CDCl}_{3}, 25^{\circ} \mathrm{C}\right): \delta=1.11(\mathrm{t}, J=$ 7.4 Hz, 3H, $\mathrm{CH}_{3}$ ethyl), 1.38-1.63 (m, 1H, $\mathrm{CH}_{2}$ ethyl), 1.87-2.06 (m, $2 \mathrm{H}, \mathrm{CH}_{2}$ ethyl, H-8), 2.33-2.48 (m, 2H, H-6, H-7), 2.94-3.03 (m, $\left.3 \mathrm{H}, 2 \mathrm{CH}_{2} \mathrm{CO}, \mathrm{H}-6\right)$, 4.06-4.22 (m, $\left.2 \mathrm{H}, \mathrm{H}-2\right), 4.08$ (s, $3 \mathrm{H}, \mathrm{NCH}_{3}$ ), 4.68 (d, $J=9.6 \mathrm{~Hz}, 1 \mathrm{H}, \mathrm{H}-8 \mathrm{a}), 4.94$ (d, $J=5.8 \mathrm{~Hz}, 1 \mathrm{H}, \mathrm{H}-3), 7.12-$ 7.43 (m, 9H, ArH, H-ind), 7.69 (d, $J=8.0 \mathrm{~Hz}, 1 \mathrm{H}, \mathrm{H}-4$ ind) ppm.

\section{(3R,7R,8S,8aR)-8-Ethyl-7-[2-(2-indolyl)-2-oxoethyl]-5-oxo-3-} phenyl-2,3,6,7,8,8a-hexahydro-5 $\mathrm{H}$-oxazolo[3,2-a]pyridine (trans-6) and its $7 S$ epimer (cis-6): Table 1, entry 4: Operating as described in the general procedure, from unsaturated lactam $\mathbf{1 a}^{[3 \mathrm{~b}, 8]}$ (400 mg, $1.64 \mathrm{mmol})$ in THF $(30 \mathrm{~mL}), \mathrm{LDA}(11 \mathrm{~mL}$ of a solution $1.5 \mathrm{M}$ in cyclohexane, $16.5 \mathrm{mmol}$ ), a solution of 2-acetylindole $\mathbf{4 b}$ $(1.3 \mathrm{~g}, 8.2 \mathrm{mmol})$ in $50 \mathrm{~mL}$ of THF for $24 \mathrm{~h}$, compound 6 was obtained as a mixture of C-7 epimers. Flash chromatography (from 1:1 to 3:7 hexane-EtOAc) afforded trans-6 (316 mg, 48\%) and cis$6(132 \mathrm{mg}, 20 \%)$. Table 1, entry 5: Operating as above, from lactam 1a (296 mg, $1.21 \mathrm{mmol})$ and acetylindole $4 \mathbf{b}$ (970 mg, 6.12 mmol) for $3 \mathrm{~h}$, pure lactams trans-6 (201 mg, $42 \%$ ) and cis-6 (150 $\mathrm{mg}, 31 \%)$ were obtained after flash chromatography. Data for trans-6: Yellow foam. $[\alpha]^{22} \mathrm{D}=-7.0\left(c=0.5, \mathrm{CHCl}_{3}\right)$. IR (film): $v=$ 1657, 1735, $3312 \mathrm{~cm}^{-1} .{ }^{1} \mathrm{H}$ NMR (300 MHz, $\left.\mathrm{CDCl}_{3}, 25^{\circ} \mathrm{C}\right): \delta=$ 1.04 (t, $J=7.2 \mathrm{~Hz}, 3 \mathrm{H}, \mathrm{CH}_{3}$ ethyl), $1.64-1.88$ (m, 3H, $\mathrm{CH}_{2}$ ethyl, $\mathrm{H}-8$ ), 2.16 (dd, $J=16.5,7.6 \mathrm{~Hz}, 1 \mathrm{H}, \mathrm{CH}_{2} \mathrm{CO}$ ), 2.57 (m, 1H, H-7), 2.68 (dd, $J=16.5,6.0 \mathrm{~Hz}, 1 \mathrm{H}, \mathrm{H}-6), 2.79$ (dd, $J=16.5,9.2 \mathrm{~Hz}, 1 \mathrm{H}$, $\mathrm{CH}_{2} \mathrm{CO}$ ), 3.00 (dd, $\left.J=16.5,4.5 \mathrm{~Hz}, 1 \mathrm{H}, \mathrm{H}-6\right), 4.10$ (dd, $J=9.0$, $1.2 \mathrm{~Hz}, 1 \mathrm{H}, \mathrm{H}-2), 4.18$ (dd, $J=9.0,6.6 \mathrm{~Hz}, 1 \mathrm{H}, \mathrm{H}-2), 4.71$ (d, $J=$ $8.1 \mathrm{~Hz}, 1 \mathrm{H}, \mathrm{H}-3), 4.98$ (d, $J=5.7 \mathrm{~Hz}, 1 \mathrm{H}, \mathrm{H}-8 \mathrm{a}), 6.87$ (d, $J=1.5$ $\mathrm{Hz}, 1 \mathrm{H}, \mathrm{H}-3 \mathrm{ind}$ ), 7.04-7.08 (m, 1H, H-6 ind), 7.18-7.25 (m, 1H, $\mathrm{H}-5$ ind), 7.28-7.41 (m, 7H, ArH, H-4 ind), 10.05 (s, 1H, NH) ppm. ${ }^{13} \mathrm{C}$ NMR $\left(75.4 \mathrm{MHz}, \mathrm{CDCl}_{3}, 25{ }^{\circ} \mathrm{C}\right): \delta=10.1\left(\mathrm{CH}_{3}\right.$ ethyl $), 22.4$ $\left(\mathrm{CH}_{2}\right.$ ethyl), $30.8(\mathrm{C}-7), 37.2\left(\mathrm{CH}_{2} \mathrm{CO}\right), 42.0(\mathrm{C}-6), 44.6(\mathrm{C}-8), 58.3$ (C-3), 73.8 (C-2), 90.7 (C-8a), 109.3 (C-3 ind), 112.5 (C-7 ind), 120.3 (C-4 ind), 122.6 (C-6 ind), 125.8 (C-5 ind), 126.7 (C-o), 127.0 (C-3a ind), 127.5 (C-m), 128.5 (C-p), 135.0 (C-7a ind), 137.7 (C-i), 141.3 (C-2 ind), $167.2 \quad$ (NCO), 190.4 (CO) $\mathrm{ppm}$. $\mathrm{C}_{25} \mathrm{H}_{26} \mathrm{~N}_{2} \mathrm{O}_{3} \cdot 1 / 4$ EtOAc (424.52): calcd. C 73.56, H 6.65, N 6.60; found C 73.77, H 6.63, N 6.47. Data for cis-6: Yellow foam. $[\alpha]^{22} \mathrm{D}$ $=-70.4\left(c=0.5, \mathrm{CHCl}_{3}\right)$. IR (film): $v=1657,1735,3325 \mathrm{~cm}^{-1} .{ }^{1} \mathrm{H}$ NMR $\left(300 \mathrm{MHz}, \mathrm{CDCl}_{3}, 25^{\circ} \mathrm{C}\right): \delta=1.10\left(\mathrm{t}, J=7.3 \mathrm{~Hz}, 3 \mathrm{H}, \mathrm{CH}_{3}\right.$ ethyl), 1.45-1.54 (m, $1 \mathrm{H}, \mathrm{CH}_{2}$ ethyl), 1.89-2.03 (m, 2H, $\mathrm{CH}_{2}$ ethyl, $\mathrm{H}-8$ ), 2.45 (m, 2H, $\mathrm{CH}_{2} \mathrm{CO}$ ), 3.00 (m, 3H, 2H-6, H-7), 4.04 (dd, $J=$ 9.3, $4.3 \mathrm{~Hz}, 1 \mathrm{H}, \mathrm{H}-2), 4.18$ (dd, $J=9.3,6.9 \mathrm{~Hz}, 1 \mathrm{H}, \mathrm{H}-2), 4.68$ (d, $J=9.3 \mathrm{~Hz}, 1 \mathrm{H}, \mathrm{H}-3), 4.94$ (d, $J=5.7 \mathrm{~Hz}, 1 \mathrm{H}, \mathrm{H}-8 \mathrm{a}), 6.87$ (d, $J=$ $1.5 \mathrm{~Hz}, 1 \mathrm{H}, \mathrm{H}-3$ ind), 7.11-7.36 (m, 9H, ArH, $\mathrm{H}$ ind), 7.68 (dd, $J=$ $8.1,0.9 \mathrm{~Hz}, 1 \mathrm{H}, \mathrm{H}-4 \mathrm{ind}), 9.50$ (s, $1 \mathrm{H}, \mathrm{NH}) \mathrm{ppm} .{ }^{13} \mathrm{C} \mathrm{NMR}(75.4$ $\left.\mathrm{MHz}, \mathrm{CDCl}_{3}, 2{ }^{\circ} \mathrm{C}\right): \delta=11.3\left(\mathrm{CH}_{3}\right.$ ethyl), $21.2\left(\mathrm{CH}_{2}\right.$ ethyl), 29.0 (C-7), $36.5\left(\mathrm{CH}_{2} \mathrm{CO}\right), 37.9$ (C-6), 43.6 (C-8), 59.4 (C-3), 73.8 (C2), 90.2 (C-8a), 109.4 (C-3 ind), 112.4 (C-7 ind), 120.8 (C-4 ind), 122.8 (C-6 ind), 126.2 (C-5 ind), 126.3 (C-o), 127.2 (C-3a ind), 127.4 (C-m), 128.4 (C-p), 135.0 (C-7a ind), 137.6 (C-i), 141.3 (C-2 ind), 166.8 (NCO), 190.7 (CO) ppm. $\mathrm{C}_{25} \mathrm{H}_{26} \mathrm{~N}_{2} \mathrm{O}_{3} \cdot 1 / 2 \quad \mathrm{H}_{2} \mathrm{O}$ (411.19): calcd. C 73.78, H 6.56, N 6.88; found C 73.40, H 6.27, N 6.98 .

(3R,6R,7R,8S,8aR)-6-(Benzyloxycarbonyl)-8-ethyl-7-[2-(1methyl-2-indolyl)-2-oxoethyl]-5-oxo-3-phenyl-2,3,6,7,8,8ahexahydro-5H-oxazolo[3,2-a]pyridine (cis-7): Operating as 
described in the general procedure, from the crude of unsaturated lactam $1 \mathbf{b}^{\text {[3a] }}(500 \mathrm{mg}, 1.32 \mathrm{mmol})$ in THF $(10 \mathrm{~mL}), \mathrm{LDA}(0.8 \mathrm{~mL}$ of a solution $2.0 \mathrm{M}$ in THF-ether, $1.6 \mathrm{mmol}$ ), and a solution of indole $4 \mathrm{a}(274 \mathrm{mg}, 1.58 \mathrm{mmol})$ in $20 \mathrm{~mL}$ of THF for $23 \mathrm{~h}$, compound 7 was obtained as a mixture of diastereoisomers. Flash chromatography (from 9:1 to 1:1 hexane-EtOAc) afforded cis-7 (228 mg, 32\%) and trans-7 (mixture of C-6 epimers; $76 \mathrm{mg}, 10 \%$ ). Data for cis-7: Yellow foam. $[\alpha]^{22} \mathrm{D}=-69.8\left(c=1.0, \mathrm{CHCl}_{3}\right)$. IR (film): $v=1660,1735 \mathrm{~cm}^{-1} .{ }^{1} \mathrm{H}$ NMR $\left(300 \mathrm{MHz}, \mathrm{CDCl}_{3}, 25^{\circ} \mathrm{C}\right): \delta$ $=1.02\left(\mathrm{t}, J=7.5 \mathrm{~Hz}, 3 \mathrm{H}, \mathrm{CH}_{3}\right.$ ethyl), $1.40-1.50$ (m, $1 \mathrm{H}, \mathrm{CH}_{2}$ ethyl), 1.85-1.94 (m, 1H, $\mathrm{CH}_{2}$ ethyl), 2.42-2.51 (m, 1H, H-8), 2.95 (dd, $J=$ $\left.16.2,11.1 \mathrm{~Hz}, 1 \mathrm{H}, \mathrm{CH}_{2} \mathrm{CO}\right), 3.13(\mathrm{dd}, J=16.2,2.4 \mathrm{~Hz}, 1 \mathrm{H}$, $\mathrm{CH}_{2} \mathrm{CO}$ ), 3.15-3.19 (m, 1H, H-7), 3.45 (d, $\left.J=0.3 \mathrm{~Hz}, 1 \mathrm{H}, \mathrm{H}-6\right)$, 4.01-4.12 (m, 1H, H-2), 4.05 (s, 3H, NCH 3 ), 4.18 (dd, $J=9.0,6.9$ $\mathrm{Hz}, 1 \mathrm{H}, \mathrm{H}-2), 4.67$ (d, $J=9.6 \mathrm{~Hz}, 1 \mathrm{H}, \mathrm{H}-8 \mathrm{a}), 4.95$ (t, $J=5.7 \mathrm{~Hz}$, $1 \mathrm{H}, \mathrm{H}-3$ ), 5.06 (d, $\left.J=12.3 \mathrm{~Hz}, 1 \mathrm{H}, \mathrm{CH}_{2} \mathrm{C}_{6} \mathrm{H}_{5}\right), 5.13$ (d, $J=12.3$ $\mathrm{Hz}, 1 \mathrm{H}, \mathrm{CH}_{2} \mathrm{C}_{6} \mathrm{H}_{5}$ ), 7.15-7.40 (m, 14H, ArH, H-ind), 7.68 (dd, $J=$ $7.8 \mathrm{~Hz}, 1 \mathrm{H}, \mathrm{H}$-ind) ppm. ${ }^{13} \mathrm{C} \mathrm{NMR}\left(75.4 \mathrm{MHz}, \mathrm{CDCl}_{3}, 25^{\circ} \mathrm{C}\right): \delta=$ $11.1\left(\mathrm{CH}_{3}\right.$ ethyl), 20.8 ( $\mathrm{CH}_{2}$ ethyl), $32.2\left(\mathrm{NCH}_{3}\right), 33.1$ (C-7), 37.4 $\left(\mathrm{CH}_{2} \mathrm{CO}\right), 40.2(\mathrm{C}-8), 53.4(\mathrm{C}-6), 59.7(\mathrm{C}-3), 66.9\left(\mathrm{CH}_{2} \mathrm{C}_{6} \mathrm{H}_{5}\right), 74.0$ (C-2), 90.3 (C-8a), 110.5 (C-3 ind), 114.4 (C-7 ind), 120.9 (C-5 ind), 122.9 (C-4 ind), 125.6 (CAr), 126.3 (CHAr), 126.4 (CHAr), 127.5 (CAr), 127.8 (CHAr), 128.0 (CAr), 128.4 (CHAr), 128.5 (CHAr), 134.5 (C-i), 135.6 (C-2 ind), 140.3 (C-3a ind), 140.6 (C$7 \mathrm{a}$ ind), 162.1 (NCO), 169.5 (COO), 190.8 (CO) ppm. $\mathrm{C}_{34} \mathrm{H}_{34} \mathrm{~N}_{2} \mathrm{O}_{5} \cdot 1 / 3$ EtOAc (579.73): calcd. C 73.18, H 6.37, N 4.83; found C 72.97, H 6.24, N 5.06.

(3R,6S,7R,8S,8aR)-8-Ethyl-7-[2-(2-indolyl)-2-oxoethyl]-6(metoxycarbonyl)-5-oxo-3-phenyl-2,3,6,7,8,8a-hexahydro-5 $\mathrm{H}$ oxazolo[3,2-a]pyridine (cis-8) and its $6 R, 7 \mathrm{~S}$ diastereoisomer (trans-8): Operating as described in the general procedure, from the crude of unsaturated lactam $1 \mathrm{c}^{[3 \mathrm{a}]}(657 \mathrm{mg}, 2.18 \mathrm{mmol})$ in THF $(10 \mathrm{~mL})$, LDA (3.84 $\mathrm{mL}$ of a solution $1.5 \mathrm{M}$ in THF, $5.76 \mathrm{mmol})$, and a solution of 2-acetylindole $4 \mathbf{b}(417 \mathrm{mg}, 2.61 \mathrm{mmol})$ in $30 \mathrm{~mL}$ of THF for $16 \mathrm{~h}$, compound $\mathbf{8}$ was obtained as a mixture of diastereoisomers. Flash chromatography (hexane to 9:1 hexaneEtOAc) afforded cis-8 (290 mg, 29\%) and trans-8 (90 mg, 9\%). Data for cis-8: Yellow foam. $[\alpha]^{22} \mathrm{D}=-65.3\left(c=1.0, \mathrm{CHCl}_{3}\right)$. IR (film): $v=1655,1736,3324 \mathrm{~cm}^{-1} .{ }^{1} \mathrm{H}$ NMR $\left(300 \mathrm{MHz}, \mathrm{CDCl}_{3}, 25\right.$ $\left.{ }^{\circ} \mathrm{C}\right): \delta=1.11\left(\mathrm{t}, J=7.6 \mathrm{~Hz}, 3 \mathrm{H}, \mathrm{CH}_{3}\right.$ ethyl), $1.44-1.54\left(\mathrm{~m}, 1 \mathrm{H}, \mathrm{CH}_{2}\right.$ ethyl), 1.89-1.99 (m, 1H, CH $\mathrm{CH}_{2}$ ethyl), 2.46-2.55 (m, 1H, H-8), 2.93 (dd, $J=16.2,11.1 \mathrm{~Hz}, 1 \mathrm{H}, \mathrm{CH}_{2} \mathrm{CO}$ ), $3.12(\mathrm{dd}, J=16.2,2.7 \mathrm{~Hz}, 1 \mathrm{H}$, $\mathrm{CH}_{2} \mathrm{CO}$ ), 3.17-3.21 (m, 1H, H-7), 3.41 (d, $\left.J=10.5 \mathrm{~Hz}, 1 \mathrm{H}, \mathrm{H}-6\right)$, $3.60\left(\mathrm{~s}, 3 \mathrm{H}, \mathrm{OCH}_{3}\right), 4.06$ (dd, $\left.J=9.0,1.5 \mathrm{~Hz}, 1 \mathrm{H}, \mathrm{H}-2\right), 4.20$ (dd, $J$ $=9.0,7.2 \mathrm{~Hz}, 1 \mathrm{H}, \mathrm{H}-2), 4.68(\mathrm{~d}, J=9.9 \mathrm{~Hz}, 1 \mathrm{H}, \mathrm{H}-8 \mathrm{a}), 4.97(\mathrm{dd}, J$ $=7.2,1.5 \mathrm{~Hz}, 1 \mathrm{H}, \mathrm{H}-3), 7.13-7.40$ (m, 9H, ArH, H-ind), 7.68 (dd, $J$ $=8.1,0.9 \mathrm{~Hz}, 1 \mathrm{H}, \mathrm{H}-4$ ind $), 9.27(\mathrm{~s}, 1 \mathrm{H}, \mathrm{NH}) \mathrm{ppm} .{ }^{13} \mathrm{C} \mathrm{NMR}(75.4$ $\left.\mathrm{MHz}, \mathrm{CDCl}_{3}, 25^{\circ} \mathrm{C}\right): \delta=11.2\left(\mathrm{CH}_{3}\right.$ ethyl), $20.0\left(\mathrm{CH}_{2}\right.$ ethyl $), 33.4$ (C-7), $35.8\left(\mathrm{CH}_{2} \mathrm{CO}\right), 40.1(\mathrm{C}-8), 52.4(\mathrm{C}-6), 53.1\left(\mathrm{OCH}_{3}\right), 59.7$ (C-3), 74.0 (C-2), 90.3 (C-8a), 109.6 (C-3 ind), 112.5 (C-7 ind), 121.0 (C-4 ind), 122.9 (C-5 ind), 126.5 (C-6 ind), 126.6 (C-o), 127.3 (C-2 ind), 127.5 (C-m), 128.3 (C-p), 134.8 (C- $i), 137.7$ (C-3a ind), 140.6 (C-7a ind), 162.4 (NCO), 170.2 (COO), 190.1 (CO) ppm. $\mathrm{C}_{27} \mathrm{H}_{28} \mathrm{~N}_{2} \mathrm{O}_{5} \cdot 1 / 2$ EtOAc (504.58): calcd. C 69.03, H 6.39, N 5.55; found C 68.99, H 6.16, N 5.59. Data for trans-8: Yellow foam. $[\alpha]^{22} \mathrm{D}=+3.6\left(c=1.0, \mathrm{CHCl}_{3}\right)$. IR (film): $v=1656,1737$, $3310 \mathrm{~cm}^{-1}$. ${ }^{1} \mathrm{H}$ NMR $\left(300 \mathrm{MHz}, \mathrm{CDCl}_{3}, 25^{\circ} \mathrm{C}\right): \delta=1.11(\mathrm{t}, J=7.5$ $\mathrm{Hz}, 3 \mathrm{H}, \mathrm{CH}_{3}$ ethyl), 1.63-1.75 (m, $1 \mathrm{H}, \mathrm{CH}_{2}$ ethyl), 1.75-1.87 (m, $1 \mathrm{H}, \mathrm{CH}_{2}$ ethyl), 1.96-2.01 (m, 1H, H-8), 2.89-2.98 (m, 1H, H-7),
3.02-3.06 (m, 2H, $\mathrm{CH}_{2} \mathrm{CO}$ ), 3.52 (d, $J=7.2 \mathrm{~Hz}, 1 \mathrm{H}, \mathrm{H}-6$ ), 3.69 (s, $\left.3 \mathrm{H}, \mathrm{OCH}_{3}\right), 4.12(\mathrm{dd}, J=9.3,1.2 \mathrm{~Hz}, 1 \mathrm{H}, \mathrm{H}-2), 4.21$ (dd, $J=9.3$, $6.6 \mathrm{~Hz}, 1 \mathrm{H}, \mathrm{H}-2), 4.83$ (d, $J=8.4 \mathrm{~Hz}, 1 \mathrm{H}, \mathrm{H}-8 \mathrm{a}), 4.98-5.00$ (m, $1 \mathrm{H}$, H-3), 7.01 (d, $J=1.8 \mathrm{~Hz}, 1 \mathrm{H}, \mathrm{H}-3$ ind), 7.11-7.45 (m, 8H, ArH, Hind), $7.66\left(\mathrm{~d}, J=8.1 \mathrm{~Hz}, 1 \mathrm{H}, \mathrm{H}-4\right.$ ind), $9.32(\mathrm{~s}, 1 \mathrm{H}, \mathrm{NH}) \mathrm{ppm} .{ }^{13} \mathrm{C}$ NMR (75.4 MHz, $\left.\mathrm{CDCl}_{3}, 25^{\circ} \mathrm{C}\right): \delta=10.3\left(\mathrm{CH}_{3}\right.$ ethyl), $23.1\left(\mathrm{CH}_{2}\right.$ ethyl), 33.7 (C-7), $41.1\left(\mathrm{CH}_{2} \mathrm{CO}\right), 44.7(\mathrm{C}-8), 52.6\left(\mathrm{OCH}_{3}\right), 54.9$ (C-6), 59.0 (C-3), 73.8 (C-2), 90.4 (C-8a), 109.6 (C-3 ind), 112.3 (C-7 ind), 120.9 (C-4 ind), 122.9 (C-5 ind), 126.8 (C-6 ind), 126.6 (C-o), 127.3 (C-2 ind), 127.5 (C-p), 128.5 (C-m), 134.8 (C-i), 137.6 (C-3a ind), 140.8 (C-7a ind), 162.7 (NCO), 170.0 (COO), 190.1 (CO) ppm. $\mathrm{C}_{27} \mathrm{H}_{28} \mathrm{~N}_{2} \mathrm{O}_{5} 3 / 4 \mathrm{H}_{2} \mathrm{O}$ (474.04): calcd. C 68.41, $\mathrm{H}$ 6.27, N 5.91; found C 68.12, H 6.10, N 5.64.

(3R,6R,7R,8S,8aR)-6-(Benzyloxycarbonyl)-8-ethyl-7-[2-(2indolyl)-2-oxoethyl]-5-oxo-3-phenyl-2,3,6,7,8,8a-hexahydro-5Hoxazolo[3,2-a]pyridine (cis-9) and its $6 S, 7 S$ diastereoisomer (trans-9): Operating as described in the general procedure, from the crude of unsaturated lactam $\mathbf{1} \mathbf{b}^{[3 \mathrm{a}]}(1.55 \mathrm{~g}, 4.11 \mathrm{mmol})$ in THF $(50 \mathrm{~mL})$, LDA (27.4 $\mathrm{mL}$ of a solution $1.5 \mathrm{M}$ in THF, $41.1 \mathrm{mmol})$, and a solution of 2-acetylindole $4 \mathbf{b}(3.27 \mathrm{~g}, 20.5 \mathrm{mmol})$ in $100 \mathrm{~mL}$ of THF for $5 \mathrm{~h}$, compound 9 was obtained as a mixture of diastereoisomers. Flash chromatography (hexane to 1:1 hexaneEtOAc) afforded cis-9 (accompanied by trace amounts of the C-6 epimer; $1.53 \mathrm{~g}, 69 \%)$ and trans-9 (457 mg, 21\%). Data for cis-9: Yellow foam. $[\alpha]^{22} \mathrm{D}=-87.0\left(c=0.2, \mathrm{CHCl}_{3}\right)$. IR (film): $v=1655$, $1735,3320 \mathrm{~cm}^{-1} .{ }^{1} \mathrm{H}$ NMR $\left(300 \mathrm{MHz}, \mathrm{CDCl}_{3}, 25^{\circ} \mathrm{C}\right): \delta=1.02(\mathrm{t}, J$ $=7.6 \mathrm{~Hz}, 3 \mathrm{H}, \mathrm{CH}_{3}$ ethyl $), 1.40-1.50\left(\mathrm{~m}, 1 \mathrm{H}, \mathrm{CH}_{2}\right.$ ethyl $), 1.83-1.94$ (m, 1H, $\mathrm{CH}_{2}$ ethyl), 2.42-2.51 (m, 1H, H-8), 2.92 (dd, $J=16.2$, $11.1 \mathrm{~Hz}, 1 \mathrm{H}, \mathrm{CH}_{2} \mathrm{CO}$ ), 3.10 (dd, $J=16.2,2.7 \mathrm{~Hz}, 1 \mathrm{H}, \mathrm{CH}_{2} \mathrm{CO}$ ), 3.16 (m, 1H, H-7), 3.47 (d, $J=1.2 \mathrm{~Hz}, 1 \mathrm{H}, \mathrm{H}-6), 4.03$ (dd, $J=9.0$, $1.5 \mathrm{~Hz}, 1 \mathrm{H}, \mathrm{H}-2), 4.19$ (dd, $J=9.0,7.2 \mathrm{~Hz}, 1 \mathrm{H}, \mathrm{H}-2), 4.67$ (d, $J=$ $9.6 \mathrm{~Hz}, 1 \mathrm{H}, \mathrm{H}-8 \mathrm{a}), 4.97$ (dd, $J=7.2,1.5 \mathrm{~Hz}, 1 \mathrm{H}, \mathrm{H}-3), 5.03$ (d, $J=$ $\left.16.8 \mathrm{~Hz}, 1 \mathrm{H}, \mathrm{CH}_{2} \mathrm{C}_{6} \mathrm{H}_{5}\right), 5.08\left(\mathrm{~d}, J=16.8 \mathrm{~Hz}, 1 \mathrm{H}, \mathrm{CH}_{2} \mathrm{C}_{6} \mathrm{H}_{5}\right), 7.12-$ 7.41 (m, 14H, ArH), 7.69 (dd, $J=8.4,1.2 \mathrm{~Hz}, 1 \mathrm{H}, \mathrm{H}-4$ ind), 9.22 (s, $1 \mathrm{H}, \mathrm{NH}) \mathrm{ppm} .{ }^{13} \mathrm{C} \mathrm{NMR}\left(75.4 \mathrm{MHz}, \mathrm{CDCl}_{3}, 25{ }^{\circ} \mathrm{C}\right): \delta=11.1\left(\mathrm{CH}_{3}\right.$ ethyl), 20.7 ( $\mathrm{CH}_{2}$ ethyl), 33.4 (C-7), $35.8\left(\mathrm{CH}_{2} \mathrm{CO}\right), 40.1$ (C-8), 53.1 (C-6), 59.5 (C-3), $66.7\left(\mathrm{CH}_{2} \mathrm{C}_{6} \mathrm{H}_{5}\right), 73.8$ (C-2), $90.0(\mathrm{C}-8 \mathrm{a})$, 109.3 (C-3 ind), 112.6 (C-7 ind), 120.6 (C-2 ind), 122.5 (C-6 ind), 126.1 (C-5 ind), 126.2 (C-4 ind), 126.9, 127.2, 127.5, 127.7, 127.9, 128.1 (C-o, $m, p), 134.6$ (C-3a ind), 135.2 (C-7a ind), 140.5 (C-i), 137.7 (C-i), 162.2 (COO), 169.2 (NCO), 189.9 (CO) ppm. $\mathrm{C}_{33} \mathrm{H}_{32} \mathrm{~N}_{2} \mathrm{O}_{5} \cdot 1 / 4$ EtOAc (558.65): calcd. C 73.10, H 6.13, N 5.01; found C 73.08, H 6.00, N 4.99. Data for trans-9: Yellow foam. ${ }^{1} \mathrm{H}$ NMR (400 MHz, $\left.\mathrm{CDCl}_{3}, 25^{\circ} \mathrm{C}\right): \delta=0.99\left(\mathrm{t}, J=7.6 \mathrm{~Hz}, 3 \mathrm{H}, \mathrm{CH}_{3}\right.$ ethyl), 1.71-2.04 (m, 3H, $\mathrm{CH}_{2}$ ethyl, $\left.\mathrm{H}-8\right), 2.87-3.18(\mathrm{~m}, 3 \mathrm{H}$, $2 \mathrm{CH}_{2} \mathrm{CO}, \mathrm{H}-7$ ), 3.56 (d, $\left.J=5.6 \mathrm{~Hz}, 1 \mathrm{H}, \mathrm{H}-6\right), 4.01-4.20(\mathrm{~m}, 2 \mathrm{H}$, H-2), 4.71 (d, $J=8.4 \mathrm{~Hz}, 1 \mathrm{H}, \mathrm{H}-8 \mathrm{a}), 4.97$ (d, $J=6.0 \mathrm{~Hz}, 1 \mathrm{H}, \mathrm{H}-3)$, $5.09\left(\mathrm{~d}, J=11.6 \mathrm{~Hz}, 1 \mathrm{H}, \mathrm{CH}_{2} \mathrm{C}_{6} \mathrm{H}_{5}\right), 5.14(\mathrm{~d}, J=11.6 \mathrm{~Hz}, 1 \mathrm{H}$, $\left.\mathrm{CH}_{2} \mathrm{C}_{6} \mathrm{H}_{5}\right), 6.95$ (d, $J=1.6 \mathrm{~Hz}, 1 \mathrm{H}, \mathrm{H}-3$ ind), 7.12-7.41 (m, $13 \mathrm{H}$, $\mathrm{ArH}$ ), 7.65 (d, $J=7.6 \mathrm{~Hz}, 1 \mathrm{H}, \mathrm{H}-4$ ind), 9.09 (bs, 1H, NH) ppm. ${ }^{13} \mathrm{C}$ NMR (100.6 MHz, $\left.\mathrm{CDCl}_{3}, 25{ }^{\circ} \mathrm{C}\right): \delta=10.4\left(\mathrm{CH}_{3}\right.$ ethyl), 23.1 ( $\mathrm{CH}_{2}$ ethyl), 34.9 (C-7), $41.1\left(\mathrm{CH}_{2} \mathrm{CO}\right), 44.7$ (C-8), 55.0 (C-6), 58.9 (C-3), $67.3\left(\mathrm{CH}_{2} \mathrm{C}_{6} \mathrm{H}_{5}\right), 73.8$ (C-2), 90.4 (C-8a), 109.7 (C-3 ind), 112.3 (C-7 ind), 120.9 (C-2 ind), 123.0 (C-6 ind), 126.4 (C-5 ind), 126.5 (C-4 ind), 126.9-128.6 (C-o, $m, p$ ), 134.8 (C-3a ind), 137.5 (C-7a ind), 140.6 (C-i), 140.8 (C-i), 162.7 (COO), 169.9 (NCO), 190.0 (CO) ppm. 
$(3 R, 6 R, 7 R, 8 S, 8 \mathrm{a} R)-6$-(tert-Butoxycarbonyl)-8-ethyl-7-[2-(2indolyl)-2-oxoethyl]-5-oxo-3-phenyl-2,3,6,7,8,8a-hexahydro-5Hoxazolo[3,2-a]pyridine (cis-10): Operating as described in the general procedure, from crude unsaturated lactam $\mathbf{1 d}^{[6]}(1.0 \mathrm{~g}, 2.90$ $\mathrm{mmol})$ in THF $(50 \mathrm{~mL})$, LDA $(19.5 \mathrm{~mL}$ of a solution $1.5 \mathrm{M}$ in cyclohexane, $29 \mathrm{mmol})$, and a solution of 2-acetylindole $4 \mathbf{b}$ ( $2.3 \mathrm{~g}$, $14.5 \mathrm{mmol}$ ) in $100 \mathrm{~mL}$ of THF for $4 \mathrm{~h}$, compound $\mathbf{1 0}$ was obtained as a mixture of diastereoisomers. Flash chromatography (hexane to 1:1 hexane-EtOAc) afforded cis-10 (1.17 g, 80\%) and trans-10 (mixture of C-6 epimers; $230 \mathrm{mg}, 7 \%$ ). Data for cis-10: Yellow foam. $[\alpha]^{22} \mathrm{D}=-21.5\left(c=0.2, \mathrm{CHCl}_{3}\right)$. IR $(\mathrm{KBr}): v=1662,1728$, $2958 \mathrm{~cm}^{-1} .{ }^{1} \mathrm{H}$ NMR $\left(400 \mathrm{MHz}, \mathrm{CDCl}_{3}, 25{ }^{\circ} \mathrm{C}\right): \delta=1.11(\mathrm{t}, J=7.6$ $\mathrm{Hz}, 3 \mathrm{H}, \mathrm{CH}_{3}$ ethyl), 1.34 [s, 9H, $\left.\left(\mathrm{CH}_{3}\right)_{3} \mathrm{C}\right], 1.43-1.52\left(\mathrm{~m}, 1 \mathrm{H}, \mathrm{CH}_{2}\right.$ ethyl), 1.88-1.96 (m, 1H, $\mathrm{CH}_{2}$ ethyl), 2.56 (ddd, $J=13.6,9.2,4.8$ $\mathrm{Hz}, 1 \mathrm{H}, \mathrm{H}-8), 2.91$ (dd, $J=16.0,10.8 \mathrm{~Hz}, 1 \mathrm{H}, \mathrm{CH}_{2} \mathrm{CO}$ ), 3.08 (dd, $J$ $\left.=16.0,2.4 \mathrm{~Hz}, 1 \mathrm{H}, \mathrm{CH}_{2} \mathrm{CO}\right), 3.20(\mathrm{~m}, 1 \mathrm{H}, \mathrm{H}-7), 3.30$ (s, 1H, H-6), 4.04 (d, $J=8.4 \mathrm{~Hz}, 1 \mathrm{H}, \mathrm{H}-2), 4.19$ (dd, $J=8.4,7.2 \mathrm{~Hz}, 1 \mathrm{H}, \mathrm{H}-2)$, $4.66(\mathrm{~d}, J=9.6 \mathrm{~Hz}, 1 \mathrm{H}, \mathrm{H}-8 \mathrm{a}), 4.97$ (d, $J=6.4 \mathrm{~Hz}, 1 \mathrm{H}, \mathrm{H}-3), 7.12-$ 7.40 (m, 9H, ArH), 7.68 (d, $J=8.4 \mathrm{~Hz}, 1 \mathrm{H}, \mathrm{H}-4$ ind), 9.36 (bs, $1 \mathrm{H}$, $\mathrm{NH})$ ppm. ${ }^{13} \mathrm{C} \mathrm{NMR}\left(100.6 \mathrm{MHz}, \mathrm{CDCl}_{3}, 25{ }^{\circ} \mathrm{C}\right): \delta=11.2\left(\mathrm{CH}_{3}\right.$ ethyl), $20.8\left(\mathrm{CH}_{2}\right.$ ethyl), $27.8\left[\mathrm{C}\left(\mathrm{CH}_{3}\right)_{3}\right], 32.9(\mathrm{C}-7), 36.0\left(\mathrm{CH}_{2} \mathrm{CO}\right)$, 40.3 (C-8), 54.4 (C-6), 59.6 (C-3), 74.1 (C-2), 81.9 [C(CH3) 3 ], 90.3 (C-8a), 109.5 (C-3 ind), 112.3 (C-7 ind), 121.0 (C-5 ind), 123.0 (C6 ind), 126.5 (C-o), 126.6 (C-2 ind), $127.4(\mathrm{C}-m), 128.4(\mathrm{C}-p)$, 134.9 (C-3a ind), 137.5 (C-7a ind), 140.8 (C-i), 162.5 (NCO), 168.3 (COO), 190.1 (CO) ppm. HRMS calcd. for $\mathrm{C}_{30} \mathrm{H}_{35} \mathrm{~N}_{2} \mathrm{O}_{5}[\mathrm{M}$ $+\mathrm{H}]^{+}$: 503.2540; found 503.2541.

$(3 R, 6 R, 7 S, 8 R, 8 \mathrm{a} S)-6-($ Benzyloxycarbonyl)-8-ethyl-7-[2-(2indolyl)-2-oxoethyl]-5-oxo-3-phenyl-2,3,6,7,8,8a-hexahydro-5Hoxazolo[3,2-a]pyridine (cis-11): Operating as described in the general procedure, from crude unsaturated lactam 1e $(161 \mathrm{mg}$, $0.43 \mathrm{mmol})$ in THF $(10 \mathrm{~mL})$, LDA $(2.86 \mathrm{~mL}$ of a solution $1.5 \mathrm{M}$ in THF, $4.3 \mathrm{mmol}$ ), and a solution of 2-acetylindole $4 \mathbf{b}$ (342 mg, 2.15 $\mathrm{mmol}$ ) in $15 \mathrm{~mL}$ of THF for $20 \mathrm{~h}$, compound $\mathbf{1 1}$ was obtained as a mixture of diastereoisomers. Flash chromatography (9:1 hexaneEtOAc to EtOAc) afforded cis-11 (110 mg, 48\%) and trans-11 (1:1 mixture of C-6 epimers; $32 \mathrm{mg}, 14 \%)$. Data for cis-11: Yellow foam. $[\alpha]^{22} \mathrm{D}=-101.1\left(c=1.2, \mathrm{CHCl}_{3}\right)$. IR $(\mathrm{KBr}): v=1656,1735$ $\mathrm{cm}^{-1} .{ }^{1} \mathrm{H}$ NMR $\left(300 \mathrm{MHz}, \mathrm{CDCl}_{3}, 25^{\circ} \mathrm{C}\right): \delta=0.98(\mathrm{t}, J=7.5 \mathrm{~Hz}$, $3 \mathrm{H}, \mathrm{CH}_{3}$ ethyl), 1.61 (m, $1 \mathrm{H}, \mathrm{CH}_{2}$ ethyl), 1.76 (m, 1H, $\mathrm{CH}_{2}$ ethyl), 1.91 (m, 1H, H-8), 2.91 (m, 1H, H-7), 3.12 (dd, $J=5.4,2.4 \mathrm{~Hz}, 2 \mathrm{H}$, $\mathrm{CH}_{2} \mathrm{CO}$ ), 3.67 (d, $\left.J=9.3 \mathrm{~Hz}, 1 \mathrm{H}, \mathrm{H}-6\right), 3.73$ (dd, $J=9.0,7.8 \mathrm{~Hz}$, $1 \mathrm{H}, \mathrm{H}-2), 4.49$ (dd, $J=9.0,7.8 \mathrm{~Hz}, 1 \mathrm{H}, \mathrm{H}-2), 4.93$ (d, $J=8.4 \mathrm{~Hz}$, $1 \mathrm{H}, \mathrm{H}-8 \mathrm{a}), 5.04$ (d, $\left.J=12.3 \mathrm{~Hz}, 1 \mathrm{H}, \mathrm{CH}_{2} \mathrm{C}_{6} \mathrm{H}_{5}\right), 5.13$ (d, $J=12.3$ $\left.\mathrm{Hz}, 1 \mathrm{H}, \mathrm{CH}_{2} \mathrm{C}_{6} \mathrm{H}_{5}\right), 5.29(\mathrm{t}, J=7.8 \mathrm{~Hz}, 1 \mathrm{H}, \mathrm{H}-3), 7.12-7.37(\mathrm{~m}$, 14H, ArH, H-ind), 7.67 (d, $J=8.1 \mathrm{~Hz}, 1 \mathrm{H}, \mathrm{H}-4$ ind), 9.21 (bs, $1 \mathrm{H}$, $\mathrm{NH})$ ppm. ${ }^{13} \mathrm{C}$ NMR $\left(75.4 \mathrm{MHz}, \mathrm{CDCl}_{3}, 25{ }^{\circ} \mathrm{C}\right): \delta=9.9\left(\mathrm{CH}_{3}\right.$ ethyl), $21.5\left(\mathrm{CH}_{2}\right.$ ethyl), $33.4(\mathrm{C}-7), 38.6\left(\mathrm{CH}_{2} \mathrm{CO}\right), 40.3(\mathrm{C}-8)$, 55.4 (C-6), 58.7 (C-3), $67.4\left(\mathrm{CH}_{2} \mathrm{C}_{6} \mathrm{H}_{5}\right), 72.6$ (C-2), 90.6 (C-8a), 109.4 (C-3 ind), 112.3 (CHind), 121.0 (CHind), 123.1 (CHind), 125.7 (CHAr), 126.5 (CHAr), 127.4 (C-i), 127.6 (CHAr), 128.0 (CHAr), 128.2 (CHAr), 128.4 (CHAr), 128.8 (CHAr), 134.8 (C-i), 135.2 (C-2 ind), 137.4 (C-3a ind), 138.6 (C-7a), 164.1 (NCO), 170.1 (COO), 190.0 (CO) ppm. $\mathrm{C}_{33} \mathrm{H}_{32} \mathrm{~N}_{2} \mathrm{O}_{5}$ (536.62): calcd. C 73.86, H 6.01, N 5.22; found C 73.46, H 6.08, N 5.34.

[3R,6R(and 6S),7S,8S,8aS]-6-(Benzyloxycarbonyl)-8-ethyl-7-[2(2-indolyl)-2-oxoethyl]-5-oxo-3-phenyl-2,3,6,7,8,8a-hexahydro$\mathbf{5 H - o x a z o l o}[3,2-a]$ pyridine (trans-12): Operating as described in the general procedure, from the crude of unsaturated lactam $\mathbf{1 f}(1 \mathrm{~g}$, $2.65 \mathrm{mmol})$ in THF $(50 \mathrm{~mL})$, LDA $(26.5 \mathrm{~mL}$ of a solution $1 \mathrm{M}$ in THF, $26.5 \mathrm{mmol})$, and a solution of 2-acetylindole $4 \mathrm{~b}(2.11 \mathrm{~g}$, $13.25 \mathrm{mmol}$ ) in $100 \mathrm{~mL}$ of THF for $5 \mathrm{~h}$, compound $\mathbf{1 2}$ was obtained as a mixture of diastereoisomers. Flash chromatography (9:1 hexane-EtOAc to EtOAc) afforded trans-12 (9:1 mixture of C-6 epimers; $1.02 \mathrm{~g}, 72 \%$ ) and cis-12 (mixture of C-6 epimers; 211 $\mathrm{mg}, \mathbf{1 4} \%$ ). Data for trans-12 (major $6 S$-epimer): Yellow foam. IR (film): $v=1655,1736,3324 \mathrm{~cm}^{-1} .{ }^{1} \mathrm{H}$ NMR $\left(400 \mathrm{MHz}, \mathrm{CDCl}_{3}, 25\right.$ $\left.{ }^{\circ} \mathrm{C}\right): \delta=0.95\left(\mathrm{t}, J=7.2 \mathrm{~Hz}, 3 \mathrm{H}, \mathrm{CH}_{3}\right.$ ethyl), $1.26-1.41\left(\mathrm{~m}, 1 \mathrm{H}, \mathrm{CH}_{2}\right.$ ethyl), 1.64-1.71 (m, $1 \mathrm{H}, \mathrm{CH}_{2}$ ethyl), 2.23-2.31 (m, 1H, H-8), 2.983.04 (m, 1H, H-7), 3.09 (dd, $J=12.4,6.0 \mathrm{~Hz}, 1 \mathrm{H}, \mathrm{CH}_{2} \mathrm{CO}$ ), 3.13 (dd, $J=12.4,6.4 \mathrm{~Hz}, 1 \mathrm{H}, \mathrm{CH}_{2} \mathrm{CO}$ ), 3.66 (d, $J=8.8 \mathrm{~Hz}, 1 \mathrm{H}, \mathrm{H}-6$ ), $3.86(\mathrm{dd}, J=8.8,6.8 \mathrm{~Hz}, 1 \mathrm{H}, \mathrm{H}-2), 4.45(\mathrm{dd}, J=8.8,8.0 \mathrm{~Hz}, 1 \mathrm{H}$, $\mathrm{H}-2), 5.09(\mathrm{~d}, J=9.2 \mathrm{~Hz}, 1 \mathrm{H}, \mathrm{H}-8 \mathrm{a}), 5.16(\mathrm{~d}, J=12.5 \mathrm{~Hz}, 1 \mathrm{H}$, $\left.\mathrm{CH}_{2} \mathrm{C}_{6} \mathrm{H}_{5}\right), 5.19\left(\mathrm{~d}, J=12.5 \mathrm{~Hz}, 1 \mathrm{H}, \mathrm{CH}_{2} \mathrm{C}_{6} \mathrm{H}_{5}\right), 5.33(\mathrm{t}, J=7.6 \mathrm{~Hz}$, $1 \mathrm{H}, \mathrm{H}-3), 7.10$ (d, $J=1.4 \mathrm{~Hz}, 1 \mathrm{H}, \mathrm{H}-3$ ind), 7.15-7.42 (m, 13H, ArH, H-ind), 7.69 (t, $J=8.0 \mathrm{~Hz}, 1 \mathrm{H}, \mathrm{H}$-ind), 9.09 (bs, 1H, NH) ppm. ${ }^{13} \mathrm{C}$ NMR $\left(100.6 \mathrm{MHz}, \mathrm{CDCl}_{3}, 25{ }^{\circ} \mathrm{C}\right): \delta=11.3\left(\mathrm{CH}_{3}\right.$ ethyl), $19.9\left(\mathrm{CH}_{2}\right.$ ethyl), 33.1 (C-7), $40.5\left(\mathrm{CH}_{2} \mathrm{CO}\right), 42.1$ (C-8), 53.8 (C-6), 58.8 (C-3), $67.2\left(\mathrm{CH}_{2} \mathrm{C}_{6} \mathrm{H}_{5}\right), 72.3(\mathrm{C}-2), 88.0$ (C-8a), $109.5(\mathrm{C}-3$ ind), 112.2 (C-7 ind), 121.1 (C-4 ind), 123.2 (C-5 ind), 125.6 (C-6 ind), 126.3, 126.7, 127.8, 128.1, 128.3, 128.4, 128.8, (C- $o$, C- $m$, C$p, \mathrm{C}-3 \mathrm{a}$ ind), 134.7 (C-2 ind), 135.5 (C-7a ind), 139.1 (C- $i$ ), 166.0 (NCO), 169.6 (COO), 190.3 (CO) ppm. HRMS calcd. for $\mathrm{C}_{33} \mathrm{H}_{32} \mathrm{~N}_{2} \mathrm{NaO}_{5}\left[\mathrm{M}+\mathrm{Na}^{+}:\right.$559.2203; found 559.2207. Data for trans-12 (minor 6R-epimer): ${ }^{1} \mathrm{H} \mathrm{NMR}\left(400 \mathrm{MHz}, \mathrm{CDCl}_{3}, 25^{\circ} \mathrm{C}\right): \delta$ $=1.00\left(\mathrm{t}, J=7.4 \mathrm{~Hz}, 3 \mathrm{H}, \mathrm{CH}_{3}\right.$ ethyl), $1.30-1.42\left(\mathrm{~m}, 2 \mathrm{H}, \mathrm{CH}_{2}\right.$ ethyl $)$, 3.15-3.20 (m, 2H, H-7, H-8), 3.25 (dd, $J=16.0,7.2 \mathrm{~Hz}, 1 \mathrm{H}$, $\mathrm{CH}_{2} \mathrm{CO}$ ), 3.30 (dd, $J=16.0,5.6 \mathrm{~Hz}, 1 \mathrm{H}, \mathrm{CH}_{2} \mathrm{CO}$ ), 3.74 (dd, $J=8.7$, $7.6 \mathrm{~Hz}, 1 \mathrm{H}, \mathrm{H}-2), 3.78$ (d, $J=5.6 \mathrm{~Hz}, 1 \mathrm{H}, \mathrm{H}-6), 4.52$ (t, $J=8.4 \mathrm{~Hz}$, $1 \mathrm{H}, \mathrm{H}-2), 5.07\left(\mathrm{~d}, J=12.4 \mathrm{~Hz}, 1 \mathrm{H}, \mathrm{CH}_{2} \mathrm{C}_{6} \mathrm{H}_{5}\right), 5.13(\mathrm{~d}, J=12.4 \mathrm{~Hz}$, $1 \mathrm{H}, \mathrm{CH}_{2} \mathrm{C}_{6} \mathrm{H}_{5}$ ), 5.16 (masked, $1 \mathrm{H}, \mathrm{H}-8 \mathrm{a}$ ), 5.38 (t, $J=7.6 \mathrm{~Hz}, 1 \mathrm{H}$, H-3), 7.00 (d, $J=1.4 \mathrm{~Hz}, 1 \mathrm{H}, \mathrm{H}-3$ ind), 7.10-7.42 (m, 13H, ArH, $\mathrm{H}$-ind), 7.68 (t, $J=8.6 \mathrm{~Hz}, 1 \mathrm{H}, \mathrm{H}$-ind), 9.07 (bs, $1 \mathrm{H}, \mathrm{NH}) \mathrm{ppm} .{ }^{13} \mathrm{C}$ NMR (100.6 MHz, $\left.\mathrm{CDCl}_{3}, 25{ }^{\circ} \mathrm{C}\right): \delta=11.6\left(\mathrm{CH}_{3}\right.$ ethyl $), 19.5\left(\mathrm{CH}_{2}\right.$ ethyl), 32.0 (C-7), $38.4\left(\mathrm{CH}_{2} \mathrm{CO}\right), 39.6$ (C-8), 50.5 (C-6), 58.1 (C3), $67.3\left(\mathrm{CH}_{2} \mathrm{C}_{6} \mathrm{H}_{5}\right), 71.9$ (C-2), 87.6 (C-8a), 109.5 (C-3 ind), 112.2 (C-7 ind), 121.1 (C-4 ind), 123.2 (C-5 ind), 125.6 (C-6 ind), 126.6, 127.5, 128.4, 128.5, 128.6, 128.8, 128.9 (C- $o$, C- $m$, C- $p$, C-3a ind), 135.1 (C-2 ind), 134.8 (C-7a ind), 137.3 (C-i), 164.8, (NCO), 169.2 (COO), 190.1.

Conversion of cis-9 to cis-6: A solution of cis-9 (200 mg, 0.43 $\mathrm{mmol})$ in EtOAc $(10 \mathrm{~mL})$ containing $\mathrm{Pd}(\mathrm{OH})_{2}(20 \mathrm{mg})$ was hydrogenated with vigorous stirring at room temperature and atmospheric pressure for $24 \mathrm{~h}$. The catalyst was removed by filtration, and the solvent was evaporated to give an oil, which was dissolved in toluene $(30 \mathrm{~mL})$. The resulting solution was heated at reflux for $16 \mathrm{~h}$ and concentrated to dryness. The residue was chromatographed (4:1 hexane-EtOAc to EtOAc) to give cis-6 (97 $\mathrm{mg}, 65 \%$ ).

(3R,7R,8S,8aS)-8-Ethyl-7-[2-(2-indolyl)-2-oxoethyl]-5-oxo-3phenyl-2,3,6,7,8,8a-hexahydro-5 $\mathrm{H}$-oxazolo $[3,2-a]$ pyridine (14): Operating as above, from trans-12 (900 mg, $1.68 \mathrm{mmol}), 30 \%$ $\mathrm{Pd}(\mathrm{OH})_{2}(270 \mathrm{mg})$ in EtOAc $(50 \mathrm{~mL})$, and then refluxing in toluene (100 mL), compound 14 was obtained (452 mg, 67\%) after column chromatography (hexane to 1:1 hexane-EtOAc). Data for 14: Yellow foam. $[\alpha]^{22} \mathrm{D}=-21.6\left(c=0.5, \mathrm{CHCl}_{3}\right)$. IR $(\mathrm{KBr})$ : 1649 , 
$3312 \mathrm{~cm}^{-1} .{ }^{1} \mathrm{H} \mathrm{NMR}\left(400 \mathrm{MHz}, \mathrm{CDCl}_{3}, 25^{\circ} \mathrm{C}\right): \delta=1.01(\mathrm{t}, J=7.4$ $\mathrm{Hz}, 3 \mathrm{H}, \mathrm{CH}_{3}$ ethyl), 1.21-1.28 (m, $1 \mathrm{H}, \mathrm{CH}_{2}$ ethyl), 1.71-1.79 (m, $1 \mathrm{H}, \mathrm{CH}_{2}$ ethyl), 2.18 (ddd, $\left.J=14.0,6.0,4.8 \mathrm{~Hz}, 1 \mathrm{H}, \mathrm{H}-8\right), 2.36$ (dd, $\left.J=18.4,3.2 \mathrm{~Hz}, 1 \mathrm{H}, \mathrm{CH}_{2} \mathrm{CO}\right), 2.63(\mathrm{dd}, J=18.4,6.4 \mathrm{~Hz}, 1 \mathrm{H}$, $\mathrm{CH}_{2} \mathrm{CO}$ ), 2.84 (m, 1H, H-7), 3.09-3.11 (m, 2H, H-6), 3.79 (dd, $J=$ $8.8,7.6 \mathrm{~Hz}, 1 \mathrm{H}, \mathrm{H}-2), 4.48$ (t, $J=8.8 \mathrm{~Hz}, 1 \mathrm{H}, \mathrm{H}-2), 5.23$ (d, $J=4.4$ $\mathrm{Hz}, 1 \mathrm{H}, \mathrm{H}-8 \mathrm{a}), 5.30$ (t, $J=7.6 \mathrm{~Hz}, 1 \mathrm{H}, \mathrm{H}-3), 7.14-7.41(\mathrm{~m}, 9 \mathrm{H}$, ArH), 7.69 (d, $J=8.0 \mathrm{~Hz}, 1 \mathrm{H}, \mathrm{H}-4$ ind), 9.35 (bs, $1 \mathrm{H}, \mathrm{NH}) \mathrm{ppm}$. ${ }^{13} \mathrm{C}$ NMR $\left(100.6 \mathrm{MHz}, \mathrm{CDCl}_{3}, 25{ }^{\circ} \mathrm{C}\right): \delta=11.6\left(\mathrm{CH}_{3}\right.$ ethyl $), 18.7$ ( $\mathrm{CH}_{2}$ ethyl), 29.6 (C-7), 33.7 ( $\left.\mathrm{CH}_{2} \mathrm{CO}\right), 40.7$ (C-8), 41.9 (C-6), 58.3 (C-3), 72.2 (C-2), 88.0 (C-8a), 109.5 (C-3 ind), 112.3 (C-7 ind), 121.1 (C-5 ind), 123.1 (C-6 ind), 126.0 (C-o), 126.7 (C-2 ind), 127.7 (C-m), 128.9 (C-p), 134.9 (C-3a ind), 137.5 (C-7a ind), 139.6 (C-i), 168.1 (NCO), 190.7 (CO) ppm. HRMS cald. for $\mathrm{C}_{25} \mathrm{H}_{27} \mathrm{~N}_{2} \mathrm{O}_{3}$ $[\mathrm{M}+\mathrm{H}]^{+}$: 403.2016; found: 403.2014.

\section{$(3 R, 7 R, 8 R, 8 \mathrm{a} S)$-8-Ethyl-7-[2-(2-indolyl)-2-oxoethyl)]-5-oxo-3-} phenyl-2,3,6,7,8,8a-hexahydro-5H-oxazolo[3,2-a]pyridine (15): Operating as above, from cis-11 (620 mg, $1.15 \mathrm{mmol}), 20 \%$ $\mathrm{Pd}(\mathrm{OH})_{2}(124 \mathrm{mg})$ in $\mathrm{MeOH}(40 \mathrm{~mL})$, and then refluxing in toluene $(70 \mathrm{~mL})$, compound 15 was obtained $(288 \mathrm{mg}, 62 \%)$ after column chromatography (hexane to $1: 1$ hexane-EtOAc). Data for 15: Yellow foam. $[\alpha]^{22} \mathrm{D}=-74.7\left(c=0.67, \mathrm{CHCl}_{3}\right)$. IR $(\mathrm{KBr}): v=$ $1648 \mathrm{~cm}^{-1} .{ }^{1} \mathrm{H}$ NMR $\left(400 \mathrm{MHz}, \mathrm{CDCl}_{3}, 25^{\circ} \mathrm{C}\right): \delta=1.08(\mathrm{t}, J=$ $7.6 \mathrm{~Hz}, 3 \mathrm{H}, \mathrm{CH}_{3}$ ethyl), 1.45-1.54 (m, 1H, $\mathrm{CH}_{2}$ ethyl), 1.75-1.90 (m, $2 \mathrm{H}, \mathrm{CH}_{2}$ ethyl, H-8), 2.54-2.66 (m, 2H, H-6, H-7), 2.90 (m, 1H, H6), $2.97\left(\mathrm{~d}, J=11.8 \mathrm{~Hz}, 1 \mathrm{H}, \mathrm{CH}_{2} \mathrm{CO}\right), 3.08(\mathrm{~d}, J=11.8 \mathrm{~Hz}, 1 \mathrm{H}$, $\mathrm{CH}_{2} \mathrm{CO}$ ), 3.80 (t, $\left.J=8.4 \mathrm{~Hz}, 1 \mathrm{H}, \mathrm{H}-2\right), 4.56(\mathrm{t}, J=8.4 \mathrm{~Hz}, 1 \mathrm{H}, \mathrm{H}-$ 2), 4.78 (d, $J=9.2 \mathrm{~Hz}, 1 \mathrm{H}, \mathrm{H}-8 \mathrm{a}), 5.35$ (t, $J=8.0 \mathrm{~Hz}, 1 \mathrm{H}, \mathrm{H}-3)$, 7.12-7.41 (m, 9H, ArH, H ind), 7.66 (d, $J=8.4 \mathrm{~Hz}, 1 \mathrm{H}, \mathrm{H}-\mathrm{ind})$, 9.26 (bs, $1 \mathrm{H}, \mathrm{NH}) \mathrm{ppm} .{ }^{13} \mathrm{C} \mathrm{NMR}\left(100.6 \mathrm{MHz}, \mathrm{CDCl}_{3}, 25{ }^{\circ} \mathrm{C}\right): \delta=$ $11.5\left(\mathrm{CH}_{3}\right.$ ethyl), $22.1\left(\mathrm{CH}_{2}\right.$ ethyl), $29.0(\mathrm{C}-7), 36.1\left(\mathrm{CH}_{2} \mathrm{CO}\right), 37.4$ (C-6), 44.0 (C-8), 58.3 (C-3), 72.4 (C-2), 90.9 (C-8a), 109.4 (C-3 ind), 112.3 (C-7 ind), 121.1 (C-4 ind), 123.0 (C-5 ind), 125.9 (C-o), 126.7 (C-6 ind), 127.4 (C-2 ind), 127.8 (C-m), 129.0 (C- $p$ ), 135.0 (C-i), 137.5 (C-3a), 139.7 (C-7a), 168.3 (NCO), 190.9 (CO) ppm. HRMS calcd. for $\mathrm{C}_{25} \mathrm{H}_{27} \mathrm{~N}_{2} \mathrm{O}_{3}[\mathrm{M}+\mathrm{H}]^{+}$: 403.2016; found: 403.2018. $\mathrm{C}_{25} \mathrm{H}_{26} \mathrm{~N}_{2} \mathrm{O}_{3} \cdot 1 / 2 \mathrm{H}_{2} \mathrm{O}$ (411.50): calcd. C 72.97, H 6.61, N 6.81; found C 72.94, H 6.61, N 6.45.

(3R,7R,8S,8aR)-8-Ethyl-7-[2-hydroxy-2-(2-indolyl)ethyl]-5-oxo3-phenyl-2,3,6,7,8,8a-hexahydro-5 $\mathrm{H}$-oxazolo[3,2-a]pyridine

(16): $\mathrm{NaBH}_{4}(117 \mathrm{mg}, 3.11 \mathrm{mmol})$ was slowly added to a solution of trans-6 (128 mg, $0.31 \mathrm{mmol})$ in $\mathrm{MeOH}(11 \mathrm{~mL})$ at $0{ }^{\circ} \mathrm{C}$. The mixture was stirred at $0{ }^{\circ} \mathrm{C}$ for $1 \mathrm{~h}$ and the temperature was slowly raised to $25^{\circ} \mathrm{C}$. The mixture was concentrated, water was added, and the aqueous layer was extracted with $\mathrm{Et}_{2} \mathrm{O}$. The combined organic extracts were dried and concentrated to give a foam, which was chromatographed (1:1 hexane-EtOAc to EtOAc) to afford alcohol $\mathbf{1 6}$ as a mixture of epimers (115 mg, 90\%). Data for $\mathbf{1 6}$ (higher $\mathrm{R}_{f}$ epimer): Yellow foam. ${ }^{1} \mathrm{H}$ NMR $\left(400 \mathrm{MHz}, \mathrm{CDCl}_{3}, 25\right.$ $\left.{ }^{\circ} \mathrm{C}\right): \delta=0.80\left(\mathrm{t}, J=7.4 \mathrm{~Hz}, 3 \mathrm{H}, \mathrm{CH}_{3}\right.$ ethyl), $1.37-1.62\left(\mathrm{~m}, 5 \mathrm{H}, \mathrm{CH}_{2}\right.$ ethyl, $\mathrm{CH}_{2} \mathrm{CHOH}, \mathrm{H}-7, \mathrm{H}-8$ ), 1.88 (dd, $J=16.8,7.2 \mathrm{~Hz}, 1 \mathrm{H}, \mathrm{H}-6$ ), $1.97\left(\mathrm{dd}, J=10.4,8.0 \mathrm{~Hz}, 1 \mathrm{H}, \mathrm{CH}_{2} \mathrm{CHOH}\right), 2.22$ (bs, $\left.1 \mathrm{H}, \mathrm{OH}\right)$, $2.43(\mathrm{dd}, J=16.8,4.4 \mathrm{~Hz}, 1 \mathrm{H}, \mathrm{H}-6), 3.92(\mathrm{dd}, J=8.8,1.6 \mathrm{~Hz}, 1 \mathrm{H}$, H-2), 3.96 (dd, $J=8.8,6.0 \mathrm{~Hz}, 1 \mathrm{H}, \mathrm{H}-2), 4.20$ (d, $J=7.6 \mathrm{~Hz}, 1 \mathrm{H}$, H-8a), 4.59 (m, 1H, CHOH), 4.77 (d, J = 5.2 Hz, 1H, H-3), 6.20 (s, $1 \mathrm{H}, \mathrm{H}-3 \mathrm{ind}), 7.02-7.27$ (m, 8H, ArH, H-ind), 7.50 (d, $J=7.2 \mathrm{~Hz}$, $1 \mathrm{H}, \mathrm{H}-4$ ind), 9.16 (bs, $1 \mathrm{H}, \mathrm{NH}) \mathrm{ppm} .{ }^{13} \mathrm{C} \mathrm{NMR}(100.6 \mathrm{MHz}$, $\left.\mathrm{CDCl}_{3}, 25{ }^{\circ} \mathrm{C}\right): \delta=9.5\left(\mathrm{CH}_{3}\right.$ ethyl), $21.2\left(\mathrm{CH}_{2}\right.$ ethyl), $31.2(\mathrm{C}-7)$,
37.4 (C-6), 40.5 ( $\left.\mathrm{CH}_{2} \mathrm{CHOH}\right), 44.4$ (C-8), 58.7 (C-3), 66.6 (CHOH), 73.7 (C-2), 90.5 (C-8a), 98.9 (C-3 ind), 111.3 (C-7 ind), 119.6 (C-5 ind), 120.3 (C-4 ind), 121.7 (C-6 ind), 126.5 (C-o), 127.6 (C-p), 128.0 (C-2 ind), 128.5 (C-m), 136.0 (C-3a ind), 140.8 (C-7a ind), 141.2 (C-i), 167.6 (NCO) ppm. Data for 16 (lower $\mathrm{R}_{f}$ epimer): Yellow foam. ${ }^{1} \mathrm{H} \mathrm{NMR}\left(400 \mathrm{MHz}, \mathrm{CDCl}_{3}, 25{ }^{\circ} \mathrm{C}\right): \delta=$ 0.91 (t, $J=7.3 \mathrm{~Hz}, 3 \mathrm{H}, \mathrm{CH}_{3}$ ethyl), 1.19-1.26 (m, 1H, $\left.\mathrm{CH}_{2} \mathrm{CHOH}\right)$, 1.41-1.45 (m, 1H, H-8), 1.49-1.59 (m, $1 \mathrm{H}, \mathrm{CH}_{2}$ ethyl), 1.68 (ddd, $J$ $=14.4,7.2,4.0 \mathrm{~Hz}, 1 \mathrm{H}, \mathrm{CH}_{2}$ ethyl), 1.88-1.92 (m, 1H, H-6), 1.952.04 (m, 2H, $\left.\mathrm{CH}_{2} \mathrm{CHOH}, \mathrm{H}-7\right), 2.21$ (bs, $\left.1 \mathrm{H}, \mathrm{OH}\right), 2.55$ (dd, $J=$ 16.4, 4.8 Hz, 1H, H-6), 3.91-3.97 (m, 2H, H-2), 4.29 (d, $J=9.2 \mathrm{~Hz}$, $1 \mathrm{H}, \mathrm{H}-8 \mathrm{a}), 4.73$ (d, $J=9.2 \mathrm{~Hz}, 1 \mathrm{H}, \mathrm{CHOH}), 4.78$ (d, $J=4.8 \mathrm{~Hz}$, 1H, H-3), 6.18 (s, 1H, H-3 ind), 7.04 (dd, $J=6.4,1.2 \mathrm{~Hz}, 1 \mathrm{H}, \mathrm{H}-7$ ind), 7.08 (dd, $J=7.6,1.6 \mathrm{~Hz}, 1 \mathrm{H}, \mathrm{H}-5$ ind), 7.09-7.24 (m, $6 \mathrm{H}$, ArH, H-6 ind), 7.53 (d, $J=8.0 \mathrm{~Hz}, 1 \mathrm{H}, \mathrm{H}-4$ ind), 9.24 (bs, $1 \mathrm{H}, \mathrm{NH}$ ) ppm. ${ }^{13} \mathrm{C}$ NMR (100.6 MHz, $\left.\mathrm{CDCl}_{3}, 25{ }^{\circ} \mathrm{C}\right): \delta=9.3\left(\mathrm{CH}_{3}\right.$ ethyl), $20.5\left(\mathrm{CH}_{2}\right.$ ethyl), 29.2 (C-7), 36.5 (C-6), $39.7\left(\mathrm{CH}_{2} \mathrm{CHOH}\right), 44.2$ (C-8), 59.0 (C-3), 64.4 (CHOH), 73.7 (C-2), 90.6 (C-8a), 97.5 (C-3 ind), 111.1 (C-7 ind), 119.4 (C-5 ind), 120.2 (C-4 ind), 121.3 (C-6 ind), 126.4 (C- $o$ ), 127.6 (C-p), 128.2 (C-2 ind), 128.6 (C-m), 135.8 (C-3a ind), 141.2 (C-7a ind), 142.4 (C-i), 167.7 (NCO) ppm.

(3R,7R,8S,8aR)-7-[2-Acetyl-2-(2-indolyl)ethyl]-8-ethyl-5-oxo-3phenyl-2,3,6,7,8,8a-hexahydro-5 $\mathrm{H}$-oxazolo[3,2-a]pyridine (17): DMAP $(8 \mathrm{mg}, 0.06 \mathrm{mmol})$ and $\mathrm{Ac}_{2} \mathrm{O}(140 \mu \mathrm{L}, 1.48 \mathrm{mmol})$ were added to a cooled $\left(0{ }^{\circ} \mathrm{C}\right)$ solution of alcohols $16(135 \mathrm{mg}, 0.34$ mmol) in $\mathrm{CH}_{2} \mathrm{Cl}_{2}$ and pyridine ( $\left.2 \mathrm{~mL}, 4: 1\right)$. The mixture was stirred at $\mathrm{rt}$ for $4 \mathrm{~h}$, diluted with $\mathrm{CHCl}_{3}$, and successively washed with $1 \mathrm{~N}$ aqueous $\mathrm{HCl}$ and saturated aqueous $\mathrm{NaHCO}_{3}$. The organic solution was dried and concentrated to afford an epimeric mixture of acetates 17 (86 mg, 57\%), which was used in the next reaction without further purification. Data for 17: Yellow foam. ${ }^{1} \mathrm{H}$ NMR (400 MHz, $\mathrm{CDCl}_{3}, 25^{\circ} \mathrm{C}$ ): $\delta=0.87$ (t, $J=7.2 \mathrm{~Hz}, 3 \mathrm{H}, \mathrm{CH}_{3}$ ethyl), 1.04 (t, $J=7.4 \mathrm{~Hz}, 3 \mathrm{H}, \mathrm{CH}_{3}$ ethyl), 1.12-1.17 (m, 2H, H-7), 1.232.21 (m, 8H, $\mathrm{CH}_{2}$ ethyl, $\left.\mathrm{CH}_{2} \mathrm{CHO}, \mathrm{H}-8\right), 2.20$ (s, 3H, $\mathrm{CH}_{3} \mathrm{CO}$ ), 2.25 (s, $\left.3 \mathrm{H}, \mathrm{CH}_{3} \mathrm{CO}\right), 2.55-2.63\left(\mathrm{~m}, 2 \mathrm{H}, \mathrm{CH}_{2} \mathrm{CHO}\right), 2.34$ (dd, $J=$ 14.0, $6.8 \mathrm{~Hz}, 1 \mathrm{H}, \mathrm{H}-6), 3.32-3.49$ (m, 3H, H-6), 4.01 (d, $J=9.0 \mathrm{~Hz}$, $1 \mathrm{H}, \mathrm{H}-2), 4.05$ (d, $J=8.8 \mathrm{~Hz}, 1 \mathrm{H}, \mathrm{H}-2), 4.07$ (d, $J=8.8 \mathrm{~Hz}, 1 \mathrm{H}, \mathrm{H}-$ 2), 4.17 (dd, $J=9.0,6.8 \mathrm{~Hz}, 1 \mathrm{H}, \mathrm{H}-2), 4.47-4.54$ (m, $3 \mathrm{H}, \mathrm{CH}_{2} \mathrm{CHO}$, H-8a), 4.70 (d, $J=8.4 \mathrm{~Hz}, 1 \mathrm{H}, \mathrm{H}-8 \mathrm{a}), 4.90$ (d, $J=6.4 \mathrm{~Hz}, 1 \mathrm{H}, \mathrm{H}-$ 3), 4.95 (d, $J=6.0 \mathrm{~Hz}, 1 \mathrm{H}, \mathrm{H}-3), 6.33$ (s, $1 \mathrm{H}, \mathrm{H}-3 \mathrm{ind}), 6.44$ (s, $1 \mathrm{H}$, $\mathrm{H}-3$ ind), 7.10 (dd, $J=14.8,7.2 \mathrm{~Hz}, 1 \mathrm{H}, \mathrm{H}-\mathrm{ind}), 7.17$ (dd, $J=14.8$, 7.2 Hz, 1H, H-ind), 7.07-7.59 (m, 18H, ArH, H-ind), 8.30 (bs, $1 \mathrm{H}$, $\mathrm{NH})$ ppm. ${ }^{13} \mathrm{C} \mathrm{NMR}\left(100.6 \mathrm{MHz}, \mathrm{CDCl}_{3}, 25{ }^{\circ} \mathrm{C}\right): \delta=9.6,9.9\left(\mathrm{CH}_{3}\right.$ ethyl), 15.2, $15.3\left(\mathrm{CH}_{2}\right.$ ethyl), 21.5, $21.6\left(\mathrm{COCH}_{3}\right), 30.3,30.9$ (C7), 36.9, 37.4 (C-6), 44.8, $44.9\left(\mathrm{CH}_{2} \mathrm{CO}\right), 58.7,58.7$ (C-3), 64.3, 64.5 (CHOAc), 73.9, 74.0 (C-2), 90.7, 91.1 (C-8a), 100.4, 102.1 (C-3 ind), 110.9, 111.0 (C-7 ind), 119.8, 119.9 (C-5 ind), 120.4, 120.5 (C-4 ind), 121.8, 122.1 (C-6 ind), 126.4, 126.5 (C-o), 127.5 (C-p), 128.0, 128.2 (C-2 ind), 128.6 (C-m), 135.9, 136.2 (C-3a ind), 137.7, 139.4 (C-3a ind), 141.2, 141.3 (C-i), 166.6 (NCO), 166.8 (COO) ppm.

\section{$(3 R, 7 R, 8 S, 8 \mathrm{a} R)-8$-Ethyl-7-[(2-indolyl)ethyl]-5-oxo-3-phenyl-} 2,3,6,7,8,8a-hexahydro-5 $H$-oxazolo $[3,2-a]$ pyridine $(18)$ : A solution of acetates $17(160 \mathrm{mg}, 0.36 \mathrm{mmol})$ in EtOAc $(25 \mathrm{~mL})$ containing $20 \%$ Pd-C (17 mg) was hydrogenated at $\mathrm{rt}$ for 4 days at atmospheric pressure. The catalyst was removed by filtration, and the solvent was evaporated to give an oil. Flash chromatography (from 9:1 to 4:1 hexane-EtOAc) afforded 18 (70 mg, 50\%). Data 
for 18: Yellow foam. ${ }^{1} \mathrm{H}$ NMR (400 MHz, $\left.\mathrm{CDCl}_{3}, 25^{\circ} \mathrm{C}\right): \delta=0.99$ (t, $J=7.6 \mathrm{~Hz}, 3 \mathrm{H}, \mathrm{CH}_{3}$ ethyl), 1.63-2.18 (m, 7H, $\mathrm{CH}_{2}$ ethyl, $\mathrm{H}-7$, $\mathrm{H}-8$, ind $\mathrm{CH}_{2} \mathrm{CH}_{2}$, ind $\left.\mathrm{CH}_{2} \mathrm{CH}_{2}\right), 2.49-2.64\left(\mathrm{~m}, 1 \mathrm{H}\right.$, ind $\left.\mathrm{CH}_{2} \mathrm{CH}_{2}\right)$, $2.53(\mathrm{dd}, J=15.0,6.0 \mathrm{~Hz}, 1 \mathrm{H}, \mathrm{H}-6), 2.77$ (ddd, $J=15.0,9.6,4.8$ $\mathrm{Hz}, 1 \mathrm{H}, \mathrm{H}-6$ ), 4.06 (dd, $J=9.2,1.2 \mathrm{~Hz}, 1 \mathrm{H}, \mathrm{H}-2), 4.16$ (dd, $J=9.2$, $6.8 \mathrm{~Hz}, 1 \mathrm{H}, \mathrm{H}-2), 4.63$ (d, $J=8.4 \mathrm{~Hz}, 1 \mathrm{H}, \mathrm{H}-8 \mathrm{a}), 4.94$ (d, $J=5.6$ $\mathrm{Hz}, 1 \mathrm{H}, \mathrm{H}-3$ ), 6.20 (s, 1H, H-3 ind), 7.03-7.57 (m, 9H, ArH, H-ind), 8.22 (bs, $1 \mathrm{H}, \mathrm{NH}) \mathrm{ppm} .{ }^{13} \mathrm{C} \mathrm{NMR}\left(100.6 \mathrm{MHz}, \mathrm{CDCl}_{3}, 25^{\circ} \mathrm{C}\right): \delta=$ $10.0\left(\mathrm{CH}_{3}\right.$ ethyl), $22.0\left(\mathrm{CH}_{2}\right.$ ethyl), 24.9 (C-6), 33.2 (C-7, ind $\mathrm{CH}_{2} \mathrm{CH}_{2}$ ), 36.8 (ind $\left.\mathrm{CH}_{2}\right), 45.1(\mathrm{C}-8), 58.7(\mathrm{C}-3), 74.0(\mathrm{C}-2)$, 91.0 (C-8a), 99.5 (C-3 ind), 110.4 (C-7 ind), 119.5 (C-5 ind), 119.7 (C-4 ind), 121.0 (C-6 ind), 126.4, 126.6 (C-o), 127.6 (C-p), 128.6 (C-m), 128.7 (C-2 ind), 135.9 (C-3a ind), 138.6 (C-7a ind), 141.2 (C-i), $167.0(\mathrm{NCO}) \mathrm{ppm}$.

(3R,7S,8S,8aR)-8-Ethyl-7-[2-hydroxy-2-(2-indolyl)ethyl]-5-oxo3-phenyl-2,3,6,7,8,8a-hexahydro-5H-oxazolo[3,2-a]pyridine

(19): Operating as in the above reduction of trans-6, from a solution of cis-6 (1.05 mg, $2.60 \mathrm{mmol})$ in THF (50 mL) and $\mathrm{NaBH}_{4}$ (148 mg, $3.91 \mathrm{mmol}$ ) for $3 \mathrm{~h}$, a foam was obtained. Flash chromatography (1:1 hexane-EtOAc to EtOAc) gave two epimeric alcohols 19 (higher $\mathrm{R}_{f}$ epimer: $462 \mathrm{mg}, 44 \%$; lower $\mathrm{R}_{f}$ epimer: 308 $\mathrm{mg}, 29 \%$ ). Data for 19 (higher $\mathrm{R}_{f}$ epimer): Yellow foam. ${ }^{1} \mathrm{H}$ NMR $\left(400 \mathrm{MHz}, \mathrm{CDCl}_{3}, 25^{\circ} \mathrm{C}\right): \delta=1.03\left(\mathrm{t}, J=7.4 \mathrm{~Hz}, 3 \mathrm{H}, \mathrm{CH}_{3}\right.$ ethyl), 1.38-2.49 (m, 8H, $\mathrm{CH}_{2}$ ethyl, $\mathrm{CH}_{2} \mathrm{CHOH}, \mathrm{H}-6, \mathrm{H}-7, \mathrm{H}-8$ ), 3.14 (bs, $1 \mathrm{H}, \mathrm{OH}), 3.94-4.04$ (m, 2H, H-2), 4.50 (d, $J=8.4 \mathrm{~Hz}, 1 \mathrm{H}, \mathrm{H}-8 \mathrm{a})$, 4.81 (m, 2H, CHOH, H-3), 6.29 (s, 1H, H-3 ind), 7.07 (t, $J=7.4$ $\mathrm{Hz}, 1 \mathrm{H}, \mathrm{H}-\mathrm{ind}), 7.13$ (t, $J=7.4 \mathrm{~Hz}, 1 \mathrm{H}, \mathrm{H}-\mathrm{ind}), 7.21-7.31$ (m, 6H, ArH, H-ind), 7.54 (d, $J=7.6 \mathrm{~Hz}, 1 \mathrm{H}, \mathrm{H}-4$ ind), 8.78 (bs, $1 \mathrm{H}, \mathrm{NH}$ ) ppm. ${ }^{13} \mathrm{C}$ NMR $\left(100.6 \mathrm{MHz}, \mathrm{CDCl}_{3}, 25{ }^{\circ} \mathrm{C}\right): \delta=11.2\left(\mathrm{CH}_{3}\right.$ ethyl), $20.7\left(\mathrm{CH}_{2}\right.$ ethyl), $28.6(\mathrm{C}-7), 35.4(\mathrm{C}-6), 36.9\left(\mathrm{CH}_{2} \mathrm{CHOH}\right), 43.8$ (C-8), 59.3 (CHOH), 65.3 (C-3), 73.8 (C-2), 90.3 (C-8a), 98.1 (C-3 ind), 111.0 (C-7 ind), 119.7 (C-5 ind), 120.4 (C-4 ind), 121.7 (C-6 ind), 126.3 (C-o), 127.6 (C-p), 128.2 (C-2 ind), $128.6(\mathrm{C}-m), 135.7$ (C-3a ind), 141.4 (C-7a ind), 141.6 (C-i), 167.7 (NCO) ppm. Data for 19 (lower $\mathrm{R}_{f}$ epimer): Yellow foam. ${ }^{1} \mathrm{H}$ NMR (400 MHz, $\left.\mathrm{CDCl}_{3}, 25{ }^{\circ} \mathrm{C}\right): \delta=0.83\left(\mathrm{t}, J=7.4 \mathrm{~Hz}, 3 \mathrm{H}, \mathrm{CH}_{3}\right.$ ethyl), $1.34-2.72$ (m, 8H, $\mathrm{CH}_{2}$ ethyl, $\left.\mathrm{CH}_{2} \mathrm{CHOH}, \mathrm{H}-6, \mathrm{H}-7, \mathrm{H}-8\right), 3.87-4.00(\mathrm{~m}, 2 \mathrm{H}$, $\mathrm{H}-2), 4.51(\mathrm{~d}, J=6.8 \mathrm{~Hz}, 1 \mathrm{H}, \mathrm{H}-8 \mathrm{a}), 4.62-4.78(\mathrm{~m}, 2 \mathrm{H}, \mathrm{CHOH}, \mathrm{H}-$ 3), 6.27 (s, 1H, H-3 ind), 7.66-7.28 (m, 8H, ArH, H-ind), 7.54 (d, $J$ $=7.6 \mathrm{~Hz}, 1 \mathrm{H}, \mathrm{H}-4 \mathrm{ind}), 9.20$ (bs, $1 \mathrm{H}, \mathrm{NH}) \mathrm{ppm} .{ }^{13} \mathrm{C} \mathrm{NMR}(100.6$ $\left.\mathrm{MHz}, \mathrm{CDCl}_{3}, 25^{\circ} \mathrm{C}\right): \delta=11.2\left(\mathrm{CH}_{3}\right.$ ethyl $), 20.8\left(\mathrm{CH}_{2}\right.$ ethyl $), 29.7$ (C-7), 30.8 (C-6), $38.0\left(\mathrm{CH}_{2} \mathrm{CHOH}\right), 44.2(\mathrm{C}-8), 59.3(\mathrm{CHOH})$, 68.2 (C-3), 73.8 (C-2), 90.3 (C-8a), 99.2 (C-3 ind), 111.3 (C-7 ind), 119.6 (C-5 ind), 120.3 (C-4 ind), 121.8 (C-6 ind), 126.3 (C-o), 127.6 (C- $p$ ), 127.9 (C-2 ind), 128.6 (C- $m$ ), 136.2 (C-3a ind), 141.0 (C-7a ind), 141.4 (C-i), 170.2 (NCO) ppm.

(3R,7S,8S,8aR)-7-[2-Acetyl-2-(2-indolyl)ethyl]-8-ethyl-5-oxo-3phenyl-2,3,6,7,8,8a-hexahydro-5 $\mathrm{H}$-oxazolo[3,2-a]pyridine (20): Operating as in the above preparation of $\mathbf{1 7}$ (reaction time $14 \mathrm{~h}$ ), from alcohols 19 (770 mg, $1.90 \mathrm{mmol}$ ), DMAP (8 mg, $0.06 \mathrm{mmol}$ ), and $\mathrm{Ac}_{2} \mathrm{O}(0.8 \mathrm{~mL}, 7.61 \mathrm{mmol})$ in $\mathrm{CH}_{2} \mathrm{Cl}_{2}$ and pyridine $(12.5 \mathrm{~mL}$, 4:1), two epimeric acetates 20 (higher $\mathrm{R}_{f}$ epimer: $440 \mathrm{mg}, 52 \%$; lower $\mathrm{R}_{f}$ epimer: $300 \mathrm{mg}$, 35\%) were obtained after column chromatography (hexane to 9:1 hexane-EtOAc). Data for 20 (higher $\mathrm{R}_{f}$ epimer): Yellow foam. $[\alpha]^{22} \mathrm{D}=+47.5\left(c=1.0, \mathrm{CHCl}_{3}\right)$. IR (KBr): $v=1641,1739,3267 \mathrm{~cm}^{-1} .{ }^{1} \mathrm{H} \mathrm{NMR}\left(400 \mathrm{MHz}, \mathrm{CDCl}_{3}\right.$, $\left.25{ }^{\circ} \mathrm{C}\right): \delta=1.11\left(\mathrm{t}, J=7.3 \mathrm{~Hz}, 3 \mathrm{H}, \mathrm{CH}_{3}\right.$ ethyl), 1.54-1.63 (m, $1 \mathrm{H}$, $\mathrm{CH}_{2}$ ethyl), 1.79 (dd, $\left.J=11.2,2.8 \mathrm{~Hz}, 1 \mathrm{H}, \mathrm{H}-6\right), 1.92-1.99$ (m, $2 \mathrm{H}$,
$\mathrm{CH}_{2}$ ethyl, $\left.\mathrm{H}-8\right), 2.10$ (s, 3H, $\mathrm{CH}_{3} \mathrm{CO}$ ), 2.26-2.31 (m, 1H, H-7), 2.35-2.45 (m, 3H, CH $\left.\mathrm{CHO}_{2} \mathrm{H}-6\right), 2.54(\mathrm{~d}, J=17.6 \mathrm{~Hz}, 1 \mathrm{H}$, $\mathrm{CH}_{2} \mathrm{CHO}$ ), 3.99 (dd, $\left.J=9.2,1.2 \mathrm{~Hz}, 1 \mathrm{H}, \mathrm{H}-2\right), 4.12$ (dd, $J=9.2$, $6.8 \mathrm{~Hz}, 1 \mathrm{H}, \mathrm{H}-2), 4.62$ (d, $J=9.2 \mathrm{~Hz}, 1 \mathrm{H}, \mathrm{H}-8 \mathrm{a}), 4.89$ (d, $J=6.8$ $\mathrm{Hz}, 1 \mathrm{H}, \mathrm{H}-3), 6.03$ (dd, $J=10.8,2.4 \mathrm{~Hz}, 1 \mathrm{H}, \mathrm{CH}_{2} \mathrm{CHO}$ ), 6.49 (d, $J$ $=2.4 \mathrm{~Hz}, 1 \mathrm{H}, \mathrm{H}-3$ ind), 7.08-7.32 (m, 8H, ArH, H-ind), 7.58 (d, $J=$ $7.2 \mathrm{~Hz}, 1 \mathrm{H}, \mathrm{H}-\mathrm{ind}), 9.18$ (bs, $1 \mathrm{H}, \mathrm{NH}) \mathrm{ppm} .{ }^{13} \mathrm{C} \mathrm{NMR}(100.6 \mathrm{MHz}$, $\left.\mathrm{CDCl}_{3}, 25{ }^{\circ} \mathrm{C}\right): \delta=11.0\left(\mathrm{CH}_{3}\right.$ ethyl $), 20.6\left(\mathrm{CH}_{2}\right.$ ethyl $), 21.1$ $\left(\mathrm{OCH}_{3}\right), 28.9$ (C-7), $32.3(\mathrm{C}-6), 37.1\left(\mathrm{CH}_{2} \mathrm{CHO}\right), 43.7(\mathrm{C}-8), 59.4$ (C-3), 67.5 ( $\left.\mathrm{CH}_{2} \mathrm{CHO}\right), 73.9$ (C-2), 90.1 (C-8a), 100.2 (C-3 ind), 111.4 (C-4 ind), 119.8 (C-5 ind), 120.6 (C-4 ind), 122.3 (C-6 ind), 126.3 (C-o); 127.5, 127.6 (C-p, C-2 ind), 128.6 (C-m), 136.0 (C-3a ind), 137.0 (C-3a ind), 141.5 (C-i), 166.8 (NCO), 171.2 (COO) ppm. HRMS calcd. for $\mathrm{C}_{27} \mathrm{H}_{31} \mathrm{~N}_{2} \mathrm{O}_{4}[\mathrm{M}+\mathrm{H}]^{+}$: 447.2278; found: 447.2300. Data for 20 (lower $\mathrm{R}_{f}$ epimer): Yellow foam. $[\alpha]^{22} \mathrm{D}=+$ $4.6\left(c=1.0, \mathrm{CHCl}_{3}\right)$. IR $(\mathrm{KBr}): v=1645,1732,3261 \mathrm{~cm}^{-1} \cdot{ }^{1} \mathrm{H}$ NMR (400 MHz, $\left.\mathrm{CDCl}_{3}, 25^{\circ} \mathrm{C}\right): \delta=0.95\left(\mathrm{t}, J=7.3 \mathrm{~Hz}, 3 \mathrm{H}, \mathrm{CH}_{3}\right.$ ethyl), 1.48-1.52 (m, $1 \mathrm{H}, \mathrm{CH}_{2}$ ethyl), 1.83-1.95 (m, 3H, $\mathrm{CH}_{2}$ ethyl, $\mathrm{H}-6, \mathrm{H}-8), 2.05$ (s, 3H, $\mathrm{CH}_{3} \mathrm{CO}$ ), 2.05-2.11 (m, 1H, H-7), 2.31-2.38 (m, 1H, H-6), 2.35 (d, $\left.J=17.6,5.6 \mathrm{~Hz}, 1 \mathrm{H}, \mathrm{CH}_{2} \mathrm{CHO}\right), 2.59$ (d, $J=$ $17.6 \mathrm{~Hz}, 1 \mathrm{H}, \mathrm{CH}_{2} \mathrm{CHO}$ ), 3.40 (dd, $\left.J=8.8,1.2 \mathrm{~Hz}, 1 \mathrm{H}, \mathrm{H}-2\right), 4.13$ (dd, $J=9.2,8.8 \mathrm{~Hz}, 1 \mathrm{H}, \mathrm{H}-2), 4.65$ (d, $J=9.2 \mathrm{~Hz}, 1 \mathrm{H}, \mathrm{H}-8 \mathrm{a}), 4.92$ (d, $J=6.8 \mathrm{~Hz}, 1 \mathrm{H}, \mathrm{H}-3), 5.96$ (dd, $J=9.2,6.0 \mathrm{~Hz}, 1 \mathrm{H}, \mathrm{CH}_{2} \mathrm{CHO}$ ), $6.52(\mathrm{~d}, J=2.0 \mathrm{~Hz}, 1 \mathrm{H}, \mathrm{H}-3 \mathrm{ind}), 7.11(\mathrm{td}, J=7.6,0.8 \mathrm{~Hz}, 1 \mathrm{H}, \mathrm{H}-$ ind), 7.17-7.32 (m, 7H, ArH, H-ind), 7.59 (d, $J=8.4 \mathrm{~Hz}, 1 \mathrm{H}, \mathrm{H}-$ ind), 8.98 (bs, $1 \mathrm{H}, \mathrm{NH}) \mathrm{ppm} .{ }^{13} \mathrm{C} \mathrm{NMR}\left(100.6 \mathrm{MHz}, \mathrm{CDCl}_{3}, 25\right.$ $\left.{ }^{\circ} \mathrm{C}\right): \delta=11.4\left(\mathrm{CH}_{3}\right.$ ethyl), $20.9\left(\mathrm{CH}_{2}\right.$ ethyl), $21.2\left(\mathrm{OCH}_{3}\right), 30.2(\mathrm{C}-$ 7), 31.8 (C-6), $37.5\left(\mathrm{CH}_{2} \mathrm{CHO}\right), 44.2$ (C-8), 59.4 (C-3), 69.2 $\left(\mathrm{CH}_{2} \mathrm{CHO}\right), 73.9$ (C-2), 90.1 (C-8a), 101.0 (C-3 ind), 111.4 (C-4 ind), 120.0 (C-5 ind), 120.8 (C-4 ind), 122.6 (C-6 ind), 126.3 (C-o), 127.5, 127.6 (C-p, C-2 ind), 128.6 (C-m), 135.8 (C-3a ind), 135.9 (C-3a ind), 141.4 (C-i), 166.7 (NCO), 171.0 (COO) ppm. HRMS calcd. for $\mathrm{C}_{27} \mathrm{H}_{30} \mathrm{~N}_{2} \mathrm{NaO}_{4}[\mathrm{M}+\mathrm{Na}]^{+}$: 469.2098; found: 469.2113.

(3R,7S,8S,8aR)-8-Ethyl-7-[(2-indolyl)ethyl]-5-oxo-3-phenyl2,3,6,7,8,8a-hexahydro-5 $H$-oxazolo $[3,2-a]$ pyridine $\quad(21)$ : A solution of acetates $20(150 \mathrm{mg}, 0.34 \mathrm{mmol})$ in EtOAc $(20 \mathrm{~mL})$ containing $20 \% \mathrm{Pd}(\mathrm{OH})_{2}(30 \mathrm{mg})$ was hydrogenated at $\mathrm{rt}$ under 400 psi of pressure for 2 days. The catalyst was removed by filtration, and the solvent was evaporated to give a foam. Flash chromatography (from 9:1 to 1:1 hexane-EtOAc) afforded 21 (71 $\mathrm{mg}, 54 \%)$. Data for 21: Yellow foam. $[\alpha]^{22} \mathrm{D}=-42.5(c=0.2$, $\left.\mathrm{CHCl}_{3}\right)$. IR (KBr): $v=1642 \mathrm{~cm}^{-1} .{ }^{1} \mathrm{H}$ NMR $\left(400 \mathrm{MHz}, \mathrm{CDCl}_{3}, 25\right.$ $\left.{ }^{\circ} \mathrm{C}\right): \delta=0.98\left(\mathrm{t}, J=7.6 \mathrm{~Hz}, 3 \mathrm{H}, \mathrm{CH}_{3}\right.$ ethyl), $1.45-1.56\left(\mathrm{~m}, 2 \mathrm{H}, \mathrm{CH}_{2}\right.$ ethyl, ind $\left.\mathrm{CH}_{2} \mathrm{CH}_{2}\right), 1.82-1.93\left(\mathrm{~m}, 2 \mathrm{H}, \mathrm{CH}_{2}\right.$ ethyl, ind $\left.\mathrm{CH}_{2} \mathrm{CH}_{2}\right)$, 1.95-2.02 (m, 1H, H-8), 2.09-2.14 (m, 1H, H-7), 2.40 (dd, $J=18.0$, $5.2 \mathrm{~Hz}, 1 \mathrm{H}$, indCH 2 ), 2.52 (dd, $J=18.0,2.0 \mathrm{~Hz}, 1 \mathrm{H}$, indCH $\left.\mathrm{CH}_{2}\right), 2.66$ (ddd, $J=14.8,10.0,7.2 \mathrm{~Hz}, 1 \mathrm{H}, \mathrm{H}-6$ ), 2.95 (ddd, $J=14.8,10.0$, $4.8 \mathrm{~Hz}, 1 \mathrm{H}, \mathrm{H}-6), 3.99$ (dd, $J=9.2,1.2 \mathrm{~Hz}, 1 \mathrm{H}, \mathrm{H}-2), 4.11$ (dd, $J=$ 9.2, 7.2 Hz, 1H, H-2), 4.61 (d, 1H, $J=9.6 \mathrm{~Hz}, 1 \mathrm{H}, \mathrm{H}-8 \mathrm{a}), 4.89$ (d, $J=6.0 \mathrm{~Hz}, 1 \mathrm{H}, \mathrm{H}-3), 6.24$ (d, $J=1.2 \mathrm{~Hz}, 1 \mathrm{H}, \mathrm{H}-3 \mathrm{ind}), 7.05-7.53$ (m, 9H, ArH, H-ind), 8.07 (bs, 1H, NH) ppm. ${ }^{13} \mathrm{C}$ NMR (100.6 $\left.\mathrm{MHz}, \mathrm{CDCl}_{3}, 25^{\circ} \mathrm{C}\right): \delta=11.3\left(\mathrm{CH}_{3}\right.$ ethyl $), 20.9\left(\mathrm{CH}_{2}\right.$ ethyl $), 26.5$ (C-6), 27.5 (ind $\mathrm{CH}_{2} \mathrm{CH}_{2}$ ), 32.4 (C-7), 36.8 (ind $\mathrm{CH}_{2}$ ), 44.1 (C-8), 59.3 (C-3), 73.8 (C-2), 90.3 (C-8a), 99.9 (C-3 ind), 110.4 (C-7 ind), 119.7 (C-5 ind), 119.8 (C-4 ind), 121.2 (C-6 ind), 126.3 (C-o), 127.5 (C-p), 128.6 (C-m), 128.7 (C-2 ind), 135.9 (C-3a ind), 138.3 (C-7a ind), 141.5 (C-i), 171.5 (NCO) ppm. HRMS calcd. for $\mathrm{C}_{25} \mathrm{H}_{29} \mathrm{~N}_{2} \mathrm{O}_{2}[\mathrm{M}+\mathrm{H}]^{+}$: 389.2224; found: 389.2224 . 
$(1 R, 5 S, 13 S)$-2-[(1R)-2-Hydroxy-1-phenylethyl]-13-ethyl-3-oxo2,3,4,5,6,7-hexahydro-3H-1,5-methanoazocine[4,3-b]indole

(22): $\mathrm{TiCl}_{4}\left(1 \mathrm{~mL}\right.$ of a $1.0 \mathrm{M}$ solution in $\left.\mathrm{CH}_{2} \mathrm{Cl}_{2}, 1.0 \mathrm{mmol}\right)$ was added to a solution of 21 (38 mg, $0.10 \mathrm{mmol})$ in $\mathrm{CH}_{2} \mathrm{Cl}_{2}(2 \mathrm{~mL})$ at $\mathrm{rt}$, and the resulting mixture was heated at reflux for $24 \mathrm{~h}$. $\mathrm{TiCl}_{4}(1$ $\mathrm{mL}$ of a $1.0 \mathrm{M}$ solution in $\mathrm{CH}_{2} \mathrm{Cl}_{2}, 1.0 \mathrm{mmol}$ ) was then added and once again after a further $24 \mathrm{~h}$, and the mixture was maintained at reflux for an additional $48 \mathrm{~h}$. The mixture was poured into saturated aqueous $\mathrm{NaHCO}_{3}$ and extracted with $\mathrm{CH}_{2} \mathrm{Cl}_{2}$. The combined organic extracts were dried and concentrated, and the resulting residue was chromatographed using a cartridge containing amine functionalized silica (1:1 hexane-EtOAc to 1:1 EtOAc$\mathrm{MeOH})$ to give $22(22 \mathrm{mg}, 58 \%)$. Data for 22: Yellow foam. $[\alpha]^{22} \mathrm{D}$ $=-88.2\left(c=0.5, \mathrm{CHCl}_{3}\right)$. IR $(\mathrm{KBr}): v=1608,2926 \mathrm{~cm}^{-1} .{ }^{1} \mathrm{H} \mathrm{NMR}$ $\left(500 \mathrm{MHz}, \mathrm{CDCl}_{3}, 25^{\circ} \mathrm{C}\right): \delta=0.72\left(\mathrm{t}, J=7.5 \mathrm{~Hz}, 3 \mathrm{H}, \mathrm{CH}_{3}\right.$ ethyl), $1.11\left(\mathrm{td}, J=7.5,7.5,2.0 \mathrm{~Hz}, 2 \mathrm{H}, \mathrm{CH}_{2}\right.$ ethyl), 1.73 (ddd, $J=14.0$, 7.0, 5.0 Hz, 1H, H-6), 1.95 (dt, $J=14.0,2.5 \mathrm{~Hz}, 1 \mathrm{H}, \mathrm{H}-6$ ), $2.05-$ 2.10 (m, 1H, H-13), 2.42-2.50 (m, 1H, H-5), 2.55 (ddd, $J=16.0$, $5.0,3.0 \mathrm{~Hz}, 1 \mathrm{H}, \mathrm{H}-7), 2.75$ (d, $J=18.5 \mathrm{~Hz}, 1 \mathrm{H}, \mathrm{H}-4), 3.00$ (dd, $J=$ $18.5,8.5 \mathrm{~Hz}, 1 \mathrm{H}, \mathrm{H}-4), 3.12(\mathrm{~m}, 1 \mathrm{H}, \mathrm{H}-7), 3.54(\mathrm{~m}, 1 \mathrm{H}$, $\left.\mathrm{C}_{6} \mathrm{H}_{5} \mathrm{CHCH}_{2}\right), 3.80-3.83\left(\mathrm{~m}, 1 \mathrm{H}, \mathrm{C}_{6} \mathrm{H}_{5} \mathrm{CHCH}_{2}\right), 4.51(\mathrm{~d}, J=5.0 \mathrm{~Hz}$, $1 \mathrm{H}, \mathrm{H}-1), 5.74\left(\mathrm{~m}, 1 \mathrm{H}, \mathrm{C}_{6} \mathrm{H}_{5} \mathrm{CHCH}_{2}\right), 6.98-7.31$ (m, 9H, ArH, Hind), 8.03 (bs, 1H, NH) ppm. ${ }^{13} \mathrm{C} \mathrm{NMR} \mathrm{(125.9} \mathrm{MHz,} \mathrm{CDCl}_{3}, 25$ $\left.{ }^{\circ} \mathrm{C}\right): \delta=11.7\left(\mathrm{CH}_{3}\right.$ ethyl $), 23.0\left(\mathrm{CH}_{2}\right.$ ethyl), 23.4 (C-6), $26.9(\mathrm{C}-7)$, 33.8 (C-5), 38.4 (C-4), 41.7 (C-13), $53.0(\mathrm{C}-1), 60.5\left(\mathrm{C}_{6} \mathrm{H}_{5} \mathrm{CHCH}_{2}\right)$, $63.2\left(\mathrm{C}_{6} \mathrm{H}_{5} \mathrm{CHCH}_{2}\right), 110.6(\mathrm{C}-12 \mathrm{~b}), 110.8(\mathrm{C}-9), 117.4(\mathrm{C}-12)$, 119.6 (C-11), 121.0 (C-10), 127.6 (C-m), 128.1 (C-o), 128.2 (C-7a), 128.3 (C-p), 133.9 (C-12a), 136.9 (C-8a), 138.5 (C-i), 172.1 (NCO) ppm. HRMS calcd. for $\mathrm{C}_{25} \mathrm{H}_{29} \mathrm{~N}_{2} \mathrm{O}_{2}[\mathrm{M}+\mathrm{H}]^{+}:$389.2224; found: 389.2222 .

\section{Acknowledgments}

Financial support from the Ministry of Science and Innovation, Spain (Project CTQ2009-07021/BQU), and the AGAUR, Generalitat de Catalunya (Grant 2009-SGR-1111) is gratefully acknowledged. Thanks are also due to the Ministry of Education and Science (Spain) for a fellowship to B. C.

[1] For reviews, see: a) D. Romo, A. I. Meyers, Tetrahedron 1991, 47, 9503-9569; b) A. I. Meyers, G. P. Brengel, Chem. Commun. 1997, 1-8; c) M. D. Groaning, A. I. Meyers, Tetrahedron 2000, 56, $9843-$ 9873; d) C. Escolano, M. Amat, J. Bosch, Chem. Eur. J. 2006, 12, 8198-8207.

[2] M. Amat, M. Pérez, A. T. Minaglia, J. Bosch, J. Org. Chem. 2008, 73, 6920-6923.

[3] a) M. Amat, M. Pérez, N. Llor, J. Bosch, E. Lago, E. Molins, Org. Lett. 2001, 3, 611-614; b) M. Amat, M. Pérez, N. Llor, J. Bosch, Org. Lett. 2002, 4, 2787-2790.

[4] a) M. Amat, M. Pérez, A. T. Minaglia, N. Casamitjana, J. Bosch, Org. Lett. 2005, 7, 3653-3656; b) M. Amat, M. Pérez, A. T. Minaglia, B. Peretto, J. Bosch, Tetrahedron 2007, 63, 5839-5848; c) M. Amat, E. Brunaccini, B. Checa, M. Pérez, N. Llor, J. Bosch, Org. Lett. 2009, 11, 4370-4373.

[5] M. Amat, J. Bosch, J. Hidalgo, M. Cantó, M. Pérez, N. Llor, E. Molins, C. Miravitlles, M. Orozco, J. Luque, J. Org. Chem. 2000, 65 , 3074-3084

[6] M. Amat, N. Llor, B. Checa, E. Molins, J. Bosch, J. Org. Chem. 2010, 75, 178-189.

[7] M. Amat, M. Pérez, S. Proto, T. Gatti, J. Bosch, Chem. Eur. J. 2010, 16, 9438-9441.

[8] M. Amat, M. Pérez, N. Llor, C. Escolano, F. J. Luque, E. Molins, J. Bosch, J. Org. Chem. 2004, 69, 8681-8693.
[9] M. Amat, N. Llor, B. Checa, M. Pérez, J. Bosch, Tetrahedron Lett. 2007, 48, 6722-6725.

[10] For conjugate addition reactions to polycyclic unsaturated lactams, see: a) E. García, E. Lete, N. Sotomayor, J. Org. Chem. 2006, 71, 6776-6784; b) S. M. Allin, J. S. Khera, J. Witherington, M. R. J. Elsegood, Tetrahedron Lett. 2006, 47, 5737-5739; c) S. M. Allin, J. S. Khera, C. I. Thomas, J. Witherington, K. Doyle, M. R. J. Elsegood, M. Edgar, Tetrahedron Lett. 2006, 47, 1961-1964; d) C. Camarero, I. González-Temprano, E. Lete, N. Sotomayor, Synlett 2007, 11011105; e) C. Camarero, I. González-Temprano, A. Gómez-SanJuan, S. Arrasate, E. Lete, N. Sotomayor, Tetrahedron 2009, 65, 5787-5798.

[11] M. Amat, N. Llor, J. Hidalgo, J. Bosch, Tetrahedron: Asymmetry 1997, 8, 2237-2240.

[12] a) T. Fujii, M. Ohba, J. Sakaguchi, Chem. Pharm. Bull. 1987, 35, 3628-3640; b) A. Deiters, M. Pettersson, S. F. Martin, J. Org. Chem. 2006, 71, 6547-6561. See also refs. 3b, 8, and 9.

[13] For the relative configuration at the epimerizable C- 6 stereocenter, see the Experimental Section. The relative configuration at C-6 was deduced from the H-6/H-7 coupling constant of $\mathrm{H}-6$ in the NMR spectra.

[14] a) P. Deslongchamps, Stereoelectronic Effects in Organic Chemistry; Pergamon Press: Oxford, 1983, p 221; b) P. Perlmutter, Conjugate Addition Reactions in Organic Synthesis; Pergamon Press: Oxford, 1992, p 25.

[15] See refs. 2, 3a, 4b, 4c, and 5-7. For a discussion of the stereochemical outcome (kinetic vs thermodynamic control) in conjugate addition reactions to unsaturated $\delta$-lactams, see refs. $3 \mathrm{~b}, 8$, and 9 .

[16] Data for 13: Yellow foam. $[\alpha]^{22}{ }_{\mathrm{D}}=-14.4\left(c=0.35, \mathrm{CHCl}_{3}\right)$. IR $(\mathrm{KBr}): v=1635 \mathrm{~cm}^{-1} .{ }^{1} \mathrm{H}$ NMR $\left(400 \mathrm{MHz}, \mathrm{CDCl}_{3}, 25^{\circ} \mathrm{C}\right): \delta=0.95(\mathrm{t}$, $J=7.6 \mathrm{~Hz}, 3 \mathrm{H}, \mathrm{CH}_{3}$ ethyl), $1.94-1.99$ (m, $2 \mathrm{H}, \mathrm{CH}_{2}$ ethyl), 2.62 (dd, $J$ $\left.=16.0,2.2 \mathrm{~Hz}, 1 \mathrm{H}, \mathrm{CH}_{2} \mathrm{CO}\right), 2.76\left(\mathrm{dd}, J=16.0,6.0 \mathrm{~Hz}, 1 \mathrm{H}, \mathrm{CH}_{2} \mathrm{CO}\right)$, 2.76-2.82 (m, 2H, H-3), 2.89-2.95 (m, 1H, H-4), 4.11-4.17 (m, $2 \mathrm{H}$, H-2'), 5.85 (t, $\left.J=7.2 \mathrm{~Hz}, 1 \mathrm{H}, \mathrm{H}-\mathrm{1}^{\prime}\right), 5.94$ (s, 1H, H-6), 6.75 (d, $J=$ $1.6 \mathrm{~Hz}, 1 \mathrm{H}, \mathrm{H}-3$ ind), $7.25-7.42(\mathrm{~m}, 8 \mathrm{H}, \mathrm{ArH}, \mathrm{H}$ ind), 7.58 (d, $J=8.4$ $\mathrm{Hz}, 1 \mathrm{H}, \mathrm{H}-4$ ind), 9.53 (bs, 1H, NH) ppm. ${ }^{13} \mathrm{C} \mathrm{NMR}(100.6 \mathrm{MHz}$, $\left.\mathrm{CDCl}_{3}, 2{ }^{\circ} \mathrm{C}\right): \delta=12.6\left(\mathrm{CH}_{3}\right.$ ethyl), $25.5\left(\mathrm{CH}_{2}\right.$ ethyl), $31.2(\mathrm{C}-4)$, $37.3\left(\mathrm{CH}_{2} \mathrm{CO}\right), 39.5$ (C-3), 57.9 (C-1'), 62.1 (C-2'), 110.0 (C-3 ind), 112.2 (C-7 ind), 120.5 (C-6), 120.8 (C-4 ind), 123.0 (C-5 ind), 125.9 (C-5), 126.4 (C-6 ind), 127.3 (C-3a ind), 127.6 (C-o), 127.9 (C-p), 128.8 (C-m), 135.2 (C-i), 137.6 (C-2 ind), 137.7 (C-7a ind), 169.5 (NCO), 191.2 (CO) ppm. HRMS calcd. for $\mathrm{C}_{25} \mathrm{H}_{27} \mathrm{~N}_{2} \mathrm{O}_{3}[\mathrm{M}+\mathrm{H}]^{+}$: 403.2016; found: 403.2016 .

[17] For the isomerization of 3,8a-cis oxazolopiperidone lactams to the more stable 3,8a-trans isomers, see: a) M. Amat, C. Escolano, A. Gómez-Esqué, O. Lozano, N. Llor, R. Griera, E. Molins, J. Bosch, Tetrahedron: Asymmetry 2006, 17, 1581-1588; b) M. Amat, M. M. M. Santos, O. Bassas, N. Llor, C. Escolano, A. Gómez-Esqué, E. Molins, S. M. Allin, V. McKee, J. Bosch, J. Org. Chem. 2007, 72, 5193-5201; c) M. Amat, M. Pérez, A. T. Minaglia, D. Passarella, J. Bosch, Tetrahedron: Asymmetry 2008, 19, 2406-2410. See also ref. 5.

[18] For recent reviews on the chemistry of $N$-acyl iminium ions, see: a) W. N. Speckamp, M. J. Moolenaar, Tetrahedron 2000, 56, 38173856; b) B. E. Maryanoff, H.-C. Zhang, J. H. Cohen, I. J. Turchi, C. A. Maryanoff, Chem. Rev. 2004, 104, 1431-1628.

[19] For the total synthesis of ervitsine in the racemic series, see: a) M.Ll. Bennasar, B. Vidal, J. Bosch, J. Org. Chem. 1997, 62, 35973609; For the enantioselective synthesis of $N$-methylervitsine, see: b) M.-Ll. Bennasar, E. Zulaica, Y. Alonso, J. Bosch, Tetrahedron: Asymmetry 2003, 14, 469-479, c) M.-Ll. Bennasar, B. Vidal, J. Bosch, Chem. Commun. 1996, 2756-2757.

[20] Data for the 8a-epimer of cis-6: Yellow foam. $[\alpha]^{22}{ }_{\mathrm{D}}=-38.0(c=$ $\left.0.2, \mathrm{CHCl}_{3}\right)$. IR $(\mathrm{KBr}): v=1652 \mathrm{~cm}^{-1} .{ }^{1} \mathrm{H} \mathrm{NMR}\left(400 \mathrm{MHz}, \mathrm{CDCl}_{3}\right.$, $\left.25{ }^{\circ} \mathrm{C}\right): \delta=1.06\left(\mathrm{t}, J=7.2 \mathrm{~Hz}, 3 \mathrm{H}, \mathrm{CH}_{3}\right.$ ethyl), $1.16-1.83(\mathrm{~m}, 8 \mathrm{H}$, $\mathrm{CH}_{2}$ ethyl, $\mathrm{H}-6, \mathrm{H}-7, \mathrm{H}-8, \mathrm{CH}_{2} \mathrm{CO}$ ), 3.77 (t, $\left.J=8.4 \mathrm{~Hz}, 1 \mathrm{H}, \mathrm{H}-2\right)$, $4.50(\mathrm{t}, J=8.4 \mathrm{~Hz}, 1 \mathrm{H}, \mathrm{H}-2), 4.89(\mathrm{~m}, 1 \mathrm{H}, \mathrm{H}-8 \mathrm{a}), 5.22(\mathrm{~m}, 1 \mathrm{H}, \mathrm{H}-3)$, 7.15-7.43 (m, 9H, ArH, H-ind), 7.73 (d, $J=8.0 \mathrm{~Hz}, 1 \mathrm{H}, \mathrm{H}$-ind), 9.09 (bs, $1 \mathrm{H}, \mathrm{NH})$ ppm. ${ }^{13} \mathrm{C}$ NMR $\left(100.6 \mathrm{MHz}, \mathrm{CDCl}_{3}, 25{ }^{\circ} \mathrm{C}\right): \delta=10.0$ $\left(\mathrm{CH}_{3}\right.$ ethyl), $21.3\left(\mathrm{CH}_{2}\right.$ ethyl), 29.7 (C-7), $30.4\left(\mathrm{CH}_{2} \mathrm{CO}\right), 40.3$ (C-6), 41.5 (C-8), 58.3 (C-3), 72.7 (C-2), 91.2 (C-8a), 112.2 (C-H ind), $135.0(\mathrm{C}-i), 137.4$ (C-3a ind), 139.7 (C-7a ind), 167.9 (NCO), 190.8 
(CO) ppm. HRMS calcd. for $\mathrm{C}_{25} \mathrm{H}_{27} \mathrm{~N}_{2} \mathrm{O}_{3}[\mathrm{M}+\mathrm{H}]^{+}$: 403.2016; found: 403.2014.

[21] M. Andriantsiferana, R. Besselièvre, C. Riche, H.-P. Husson, Tetrahedron Letters 1977, 18, 2587-2590.

[22] For the chemoselective oxidation of a 2-indolylmethyl to a 2acylindole moiety, see: a) Y. Ban, K. Yoshida, J. Goto, T. Oishi, J. Am. Chem. Soc. 1981, 103, 6990-6992, b) Y. Ban, K. Yoshida, J.

Goto, T. Oishi, E. Takeda, Tetrahedron 1983, 39, 3657-3668, c) T. Fujii, M. Ohba, J. Sakaguchi, Chem. Pharm. Bull. 1987, 35, 36283640, d) J. Gracia, N. Casamitjana, J. Bonjoch, J. Bosch, J. Org. Chem. 1994, 59, 3939-3951.

Received: ((will be filled in by the editorial staff)) Published online: ((will be filled in by the editorial staff)) 


\section{Layout 2:}

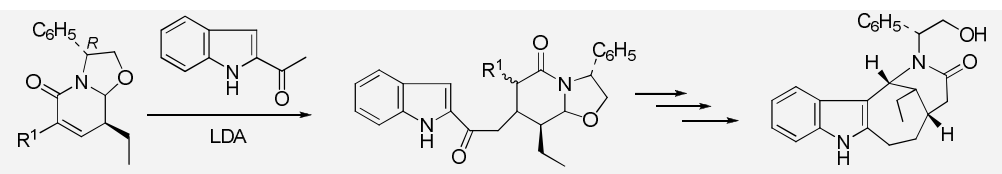

$\mathrm{R}^{1}=\mathrm{H}, \mathrm{CO}_{2} \mathrm{R}^{2}$

The stereochemical outcome of the conjugate addition reactions of 2acetylindole enolates to phenylglycinolderived unsaturated lactams is studied.
A subsequent Lewis acid-promoted intramolecular $\quad \alpha$-amidoalkylation reaction leads to the tetracyclic ring system of ervitsine.

((Key Topic))

M. Amat, ${ }^{*}$ B. Checa, N. Llor, M. Pérez, J. Bosch Page No. - Page No.

Conjugate Addition of 2-Acetylindoles to Unsaturated Oxazolopiperidone Lactams. Enantioselective Access to the Tetracyclic Ring System of Ervitsine.

Keywords: Lactams / Asymmetric synthesis / Cyclization / Michael addition / Heterocycles 
